\title{
Sugar-responsive pseudopolyrotaxanes and their application in sugar-induced release of PEGylated insulin
}

Tomohiro Seki, Keigo Abe, Kiminobu Nakamura, Yuya Egawa,* Ryotaro Miki, Kazuhiko Juni, Toshinobu Seki

Faculty of Pharmaceutical Sciences, Josai University, Keyakidai, Sakado, Saitama 350-0295, Japan.

Corresponding author. Tel.: +81 49271 7686; fax: +81 492717686.

E-mail address: yegawa@josai.ac.jp (Y. Egawa).

\begin{abstract}
We have designed a pseudopolyrotaxane (PPRX), known as a molecular necklace, consisting of phenylboronic acid-modified $\gamma$-cyclodextrin (PBA- $\gamma$-CyD) and naphthalene-modified polyethylene glycol (Naph-PEG) for developing sugar-responsive insulin delivery systems. Interestingly, structural analyses show that the Naph-PEG/PBA- $\gamma$-CyD PPRX obtained by our method was single stranded, whereas ordinary PPRXs using parent $\gamma$-CyD were double stranded. The Naph-PEG/PBA- $\gamma$-CyD PPRX was poorly water soluble at $\mathrm{pH}$ 7.4; however, sugar addition induced disintegration of the PPRX, and the components were dissolved, suggesting that the PBA moiety acts as a sugar sensor. We also have developed a PPRX consisting of Naph-PEG-appended insulin (Naph-PEG-Ins) and PBA- $\gamma$-CyD and have confirmed that the release rate of Naph-PEG-Ins was accelerated following sugar addition.
\end{abstract}

\section{Keywords}

Cyclodextrin; Pseudopolyrotaxane; Phenylboronic acid; Insulin; Polypseudorotaxane; Intelligent material 


\section{Introduction}

One of the goals of drug delivery system is to control the timing and rate of drug release. Sustained drug release systems are one of the most successful time-controlled systems, and they have been applied to not only sustained-release oral tablets [1] but also long-acting injectable microspheres [2, 3] and transdermal sustained-release systems [4, 5]. There has recently been considerable interest in stimuli-sensitive drug delivery systems that can control the timing of drug release [6-8]. Such systems require the ability to recognize the surrounding environment of systems and the mechanism for drug release. For this purpose, cyclodextrin (CyD)-based molecular machines have attracted much attention [9, 10]. CyDs are cyclic oligosaccharides composed of glucopyranoside units. CyDs consist of a hydrophobic cavity in which hydrophobic molecules are enclosed to form an inclusion complex. CyDs include not only low molecular weight molecules but also polymers. The complexes in which many CyDs are threaded by a polymer are called pseudopolyrotaxanes (PPRXs) or polypseudorotaxanes. When both end groups of a polymer of PPRX are modified with bulky stoppers, the structures are called polyrotaxanes (PRXs). Harada et al. first reported that $\alpha$-CyD forms a crystalline complex, with polyethylene glycol (PEG) in which PEG penetrated many cavities of $\alpha$-CyD [11]. After their discovery, several combinations of CyD and polymers for PPRXs have been developed [12,13]. The unique structures of PPRXs and PRXs have been applied for a new generation of drug delivery systems [14-24].

It is reasonable to use PEG for drug delivery systems because PEG itself is biocompatible and is approved as a pharmaceutical additive. Furthermore, PEGylation technologies have been widely used to improve therapeutic efficacies of some protein drugs, and some PEGylated proteins are in the market [25, 26]. Higashi et al. have prepared PPRXs composed of a PEGylated insulin (PEG-Ins) and $\alpha$-CyD or $\gamma$-CyD [22-24]. Following subcutaneous administration of the PEG-Ins/CyD PPRX, the plasma levels of PEG-Ins in rats were significantly prolonged, achieving an extended hypoglycemic effect. The results suggest that the PEG-Ins/CyD PPRX can work as a sustained drug release system. However, the PEG-Ins/CyD PPRX was not designed to control the release rate of PEG-Ins, depending on the D-glucose (Glc) concentration. Sugar-responsive insulin (Ins) release systems are highly expected to increase the degree of freedom and convenience of use of self-injections for diabetic patients because Ins self-injection treatments are associated with difficulties concerning controlled blood sugar levels and risk of hypoglycemia.

For developing sugar-responsive drug delivery systems, phenylboronic acid (PBA) constructs are promising. PBA reacts with cis-diol functional groups of sugars to form a five- or six-membered ring via ester bonds. As a result, PBA derivatives have been widely investigated as sugar-recognition motifs in chemical probes for sugar analysis [27-32] and sugar-sensitive Ins release systems [33-35]. We recently synthesized PBA-modified CyDs (PBA-CyDs) and found that they form supramolecular structures [36]. PBA- $\alpha$-CyD formed a supramolecular polymer via head-to-tail interactions, whereas PBA- $\beta-$ CyD formed a dimer via head-to-head interactions. The resulting supramolecular structures showed low solubility in water; however, the addition of sugar induced an increase in their solubility. These results suggest a new approach to chemical-responsive materials on the basis of the use of guest-modified CyDs. To progress to the next stage, we must develop a construct that combines the supramolecular structures using PBA-CyDs and Ins.

PBA- $\gamma$-CyD does not form a poorly-soluble supramolecular structure by itself because the $\gamma$-CyD cavity is too large to include the PBA moiety. On the basis of the idea that the large cavity of PBA- $\gamma-C y D$ may be suitable for the inclusion of PEG chains, we combined PBA- $\gamma$-CyD and PEG-Ins in a PPRX. The building blocks for PPRXs are shown in Fig. 1. Naphthalene-modified PEG (Naph-PEG) was used because our preliminary experiments revealed that Naph-PEG was 
more effective in forming PPRXs compared with unmodified PEG. We investigated the resulting PPRX composed of Naph-PEG and PBA- $\gamma$-CyD (Naph-PEG/PBA- $\gamma$-CyD) to identify both its structure and sugar response. Then, we applied the PPRX system to Naph-PEG-appended Ins (Naph-PEG-Ins) to fabricate a new type of sugar-responsive Ins release system (Fig. 2).

\section{Experimental}

Materials

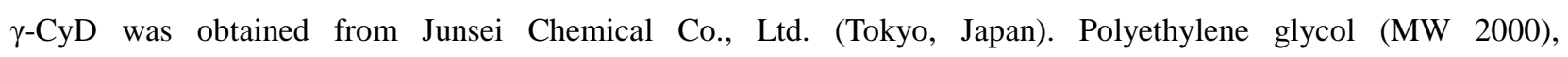
p-chlorocarbonylphenylboronic acid, and Ins (human, recombinant) were purchased from Wako Pure Chemical Industries (Osaka, Japan). 2-Naphthoyl chloride and 1-ethyl-3-(3-dimethylaminopropyl) carbodiimide hydrochloride (EDC) were obtained from Tokyo Chemical Industry Co., Ltd. (Tokyo, Japan). 2-[4-(2-Hydroxyethyl)-1-piperazinyl] ethanesulfonic acid (HEPES) was purchased from Dojindo Laboratories (Kumamoto, Japan). Sunbright PA-020HC $\left[\mathrm{H}_{2} \mathrm{~N}-\left(\mathrm{CH}_{2}\right)_{3}-\mathrm{O}-\mathrm{PEG}-\left(\mathrm{CH}_{2}\right)_{5}-\mathrm{COOH}\right.$, MW 2000] was obtained from NOF Corporation (Tokyo, Japan). All other chemicals were of reagent grade and were used as received.

Apparatus

${ }^{1} \mathrm{H}$ NMR measurements were conducted with a Varian 400-MR (Agilent Technologies, California, USA). Solid-state fluorescence spectroscopy was performed with RF-5300PC spectrofluorophotometer (Shimadzu Corporation, Kyoto, Japan). Turbidity was monitored with a V-530 UV-vis spectrometer (JASCO Corporation, Tokyo, Japan) using absorbance at $700 \mathrm{~nm}$. The release rate of Naph-PEG was monitored using a Spectra Max M5e multiplate reader (Molecular Devices Japan, Tokyo, Japan). Differential scanning calorimetry (DSC) was carried out using a Thermo Plus2 series (Rigaku Corporation, Tokyo, Japan). A sample was heated in an aluminum pan at a heating rate of $5 \mathrm{~K} / \mathrm{min}$ under a nitrogen atmosphere. Powder X-ray diffraction patterns were measured using a Mini FlexII (Rigaku Corporation, Tokyo, Japan) under the following conditions: We used $\mathrm{CuK} \alpha$ radiation, and diffraction was done at $30 \mathrm{kV}, 15 \mathrm{~mA}$ with a scanning speed of $4^{\circ} / \mathrm{min}$ and measurement range of $2 \theta=3^{\circ}-40^{\circ}$. MALDI-TOF MS spectra were recorded with AXIMA-CFR plus (Shimadzu).

Synthesis

Phenylboronic acid-modified $\gamma$-cyclodextrin $(P B A-\gamma-C y D)$

$\gamma$-CyD (2.61 g, $2.00 \mathrm{mmol}$ ) was dried under reduced pressured for $4 \mathrm{~h}$ at $100^{\circ} \mathrm{C}$, and it was dissolved into anhydrous pyridine $(200 \mathrm{~mL})$. p-Chlorocarbonylphenylboronic acid (1.00 g, $5.43 \mathrm{mmol})$ was added to the solution. The solution was stirred at $90^{\circ} \mathrm{C}$, and it was check to with silikagel TLC (Eluent, $n-\mathrm{BtOH}: \mathrm{EtOH}: \mathrm{H}_{2} \mathrm{O}=5: 4: 3$ in volume ratio, Coloring with anisaldehyde). After di-modified PBA- $\gamma$-CyD appeared, the reaction was stopped by the addition of small amount of water. The reaction solution was evaporated to concentrate, $40 \mathrm{~mL}$ of the concentrated solution was added to $400 \mathrm{~mL}$ of acetone, and the precipitate was filtered using a glass filter. The white powder was dissolved in $200 \mathrm{~mL}$ of water, and the 
solution was filtered to remove insoluble matter. The filtrate was applied to column chromatography using a highly porous polystyrene gel (Diaion HP-20, Mitsubishi Chemical, Japan). The mixed solution of $\mathrm{H}_{2} \mathrm{O}$ and $\mathrm{MeOH}$ was used as an eluent. When the volume ratio of $\mathrm{MeOH}: \mathrm{H}_{2} \mathrm{O}$ was $1: 1$, the mono modified PBA- $\gamma$-CyD was eluted. The eluates containing PBA- $\gamma$-CyD were gathered and were evaporated to concentrate. Forty $\mathrm{mL}$ of the concentrated solution was added to $400 \mathrm{~mL}$ of acetone, and the precipitate was filtered using a glass filter. The white powder was washed with acetone three times and was dried in vacuo (0.449 g, 15.5\%).

${ }^{1} \mathrm{H}$ NMR (400 MHz, DMSO-d ${ }_{6}$, Fig. S1) $\delta 8.25$ (s, 2H, BOH), 7.97-7.79 (m, 4H, ArH), 5.99-5.56 (m, 16H, СyD OH-2, OH-3), 4.97-4.80 (m, 8H, CyD H-1), 4.59-4.41 (m, 7H, CyD OH-6), 3.73-3.40 (m, 32H, CyD H-3, H-5, H-6a, b) 3.373.25 (m, overlaps with HOD).

FAB-MS, negative mode, matrix: glycerol m/z: 1499.5 [(M + glycerol - $\left.2 \mathrm{H}_{2} \mathrm{O}-\mathrm{H}\right)^{-}$requires 1499.5]. The PBA- $\gamma-\mathrm{CyD}$ was detected as an ester form composed of PBA- $\gamma-\mathrm{CyD}$ and glycerol.

Anal. Calcd for $\mathrm{C}_{55} \mathrm{H}_{85} \mathrm{BO}_{43}+6 \mathrm{H}_{2} \mathrm{O}$ : C, 42.53; H, 6.29; B, 0.70; O, 50.48. Found: C, 42.37; H, 6.06.

\section{Naphthalene-modified polyethylene glycol (Naph-PEG)}

PEG (MW 2000, $10.0 \mathrm{~g}$ ) was dissolved in a mixed solution of anhydrous dichloromethane (300 $\mathrm{mL})$ and anhydrous pyridine $(22.5 \mathrm{~mL})$. The solution was stirred at $0^{\circ} \mathrm{C}$, and 2-naphthoyl chloride $(6.4 \mathrm{~g}, 34 \mathrm{mmol})$ was added to the solution. It was stirred at $0^{\circ} \mathrm{C}$ under a nitrogen atmosphere for 3 days. A small amount of water was added to stop the reaction, and the solution was filtered with a glass filter to remove insoluble matter. The filtrate was evaporated, and the residue was recrystallized with EtOH.

${ }^{1} \mathrm{H}$ NMR (400 MHz, D $\mathrm{O}$, Fig. S2) $\delta$ 8.37-8.61 (s, 1H, Naphthoyl H-1), 7.80-7.63 (m, 4H, Naphthoyl H-3, H-4, H-5, H-8), 7.50-7.35 (m, 2H, Naphthoyl H-6, H-7), 4.38-4.23 (m, 2H, Carbonyl $\alpha-\mathrm{H}), 3.54-3.45$ [m, 85H, $\left.-\left(\mathrm{CH}_{2}-\mathrm{CH}_{2}-\mathrm{O}\right)_{\mathrm{n}}-\right]$. MALDI-TOF MS m/z: $2466.70[\mathrm{M}+\mathrm{H}]^{+}$.

One-terminal naphthalene-modified polyethylene glycol (One-Naph-PEG)

$\mathrm{H}_{2} \mathrm{~N}-\left(\mathrm{CH}_{2}\right)_{3}$-O-PEG- $\left(\mathrm{CH}_{2}\right)_{5}-\mathrm{COOH}$ (MW 2000, $300 \mathrm{mg}$ ) was dissolved in a mixed solution of anhydrous dichloromethane $(10 \mathrm{~mL})$ and anhydrous pyridine $(0.70 \mathrm{~mL})$. The solution was stirred at $0^{\circ} \mathrm{C}$, and 2-naphthoyl chloride (286 mg, $1.50 \mathrm{mmol}$ ) was added to the solution. It was stirred at $0^{\circ} \mathrm{C}$ under a nitrogen atmosphere for 4 days. The solvent was evaporated, and water $(40 \mathrm{~mL})$ was added to the residue. The solution was filtered with glass filter to remove insoluble matter, and the filtrate was dialyzed against water using a dialysis tube (MWCO 1000). The resulting solution was lyophilized, and One-Naph-PEG was obtained (229 mg, $69.3 \%$ )

${ }^{1} \mathrm{H}$ NMR (400 MHz, D $\mathrm{O}$, Fig. S3) $\delta$ 8.22-8.16 (s, 1H, Naphthoyl H-1), 7.93-7.81 (m, 3H, Naphthoyl H-3, H-4, H-5), 7.69-7.64 (d, 1H, Naphthoyl H-8), 7.55-7.45 (m, 2H, Naphthoyl H-6, H-7), 3.72-3.66 (m, 2H, $\alpha-\mathrm{H}$ of the amide group), 3.53-3.51 [m, 187H, - $\left(\mathrm{CH}_{2}-\mathrm{CH}_{2}-\mathrm{O}\right)_{\mathrm{n}}$ ] $], 2.03-1.97$ (t, $2 \mathrm{H}, \alpha-\mathrm{H}$ of the carboxyl group), 1.82-1.74 (m, 2H, alkyl), 1.65-1.56 (m, 2H, alkyl), 1.47-1.34 (m, 8H, alkyl).

MALDI-TOF MS m/z: $2394.46[\mathrm{M}+\mathrm{H}]^{+}$. 
Ins $(208 \mathrm{mg}, 48.2 \mu \mathrm{mol})$ and One-Naph-PEG (0.208 g, $96.3 \mu \mathrm{mol})$ were dissolved in DMSO (28 mL), and EDC (18.0 mg, $96.3 \mu \mathrm{mol})$ was added. The solution was stirred at room temperature for $48 \mathrm{~h}$, and water $(80 \mathrm{~mL})$ was added. The solution was dialyzed against water using a dialysis tube (MWCO 3500). The resulting solution was lyophilized, and Naph-PEG-Ins was obtained (348 mg, 66.3\%). The stoichiometry of PEG/Ins in the Naph-PEG-Ins was calculated to be 2.2. The details are described in the Supplementary Data (Figs. S4 and S5).

Preparation of PPRXs

Naph-PEG/PBA- $\gamma-C y D P P R X$

Naph-PEG (12.3 mg, $5.5 \mu \mathrm{mol})$ and PBA- $\gamma$-CyD (100 mg, $69.2 \mu \mathrm{mol})$ were dissolved in water $(500 \mu \mathrm{L})$, and the solution was kept at room temperature. After 28 days, the resulting precipitate of the PPRX was filtered and dried under reduced pressure $(84.7 \mathrm{mg})$. The Naph-PEG/PBA- $\gamma$-CyD PPRX was obtained as a crystalline precipitate but not a gel (Fig. S6). The Naph-PEG/PBA- $\gamma-C y D$ PPRX was dissolved in DMSO- $d_{6}$ and measured with ${ }^{1} \mathrm{H}$ NMR (Fig. S7). When the integration of H-1 of CyD was set to be 8.00, the integration of the PEG part was 8.10. From this result, we calculated the stoichiometry between the ethylene glycol monomer unit and PBA- $\gamma-C y D$ to be 2.1:1.0.

Naph-PEG-Ins/PBA- $\gamma-C y D P P R X$

Naph-PEG-Ins (33.4 mg, $30.8 \mu \mathrm{mol})$ and PBA- $\gamma$-CyD (200 mg, $138.4 \mu \mathrm{mol})$ were dissolved in water $(1000 \mu \mathrm{L})$, and the solution was kept at room temperature. After 28 days, the resulting precipitate of the PPRX was filtered and dried under reduced pressure (117.2 mg). The Naph-PEG-Ins/PBA- $\gamma$-CyD PPRX was formed as a crystalline precipitate but not a gel (Fig. S8). The Naph-PEG/PBA- $\gamma$-CyD PPRX was dissolved in DMSO- $d_{6}$ and measured with ${ }^{1} \mathrm{H}$ NMR (Fig. S9). When the integration of H-1 of CyD was set to be 8.00, the integration of the PEG part was 9.38. From this result, we calculated the stoichiometry between the ethylene glycol monomer unit and PBA- $\gamma$-CyD to be 1.4:1.0.

Evaluation of sugar responsiveness of PPRXs

\section{Turbidity measurements}

A buffer solution (20 mM HEPES, pH 7.4, $2.0 \mathrm{~mL}$ ) was added to a cell for absorption spectrometer, and the buffer solution was kept at $37^{\circ} \mathrm{C}$ and stirred. The Naph-PEG/PBA- $\gamma$-CyD PPRX (6.0 mg) was suspended in the stirred buffer solution, and the turbidity was measured by absorbance at $700 \mathrm{~nm}$. After the turbidity became constant, a small amount of stock sugar solution $(1.00 \mathrm{M})$ was added at 10-min intervals to the suspended solution to increase the sugar concentration.

Release tests of PEG chains using fluorescence of the naphthoyl moiety 
A buffer solution with and without sugars (20 mM HEPES, pH 7.4, $1.0 \mathrm{~mL}$ ) was added to a microtube for centrifugation, and the buffer solution was kept at $37^{\circ} \mathrm{C}$. A PPRX (Naph-PEG/PBA- $\gamma$-CyD PPRX: 3.0 mg, Naph-PEG-Ins/PBA- $\gamma$-CyD PPRX: $6.0 \mathrm{mg}$ ) was added to the solution. After a certain time, the microtube was centrifuged, and the small amount of supernatant was collected. The fluorescence derived from the naphthoyl moiety was monitored with a microplate reader $\left(\lambda_{\mathrm{ex}}=280 \mathrm{~nm}, \lambda_{\mathrm{em}}=420 \mathrm{~nm}\right)$.

\section{Results and discussion}

Preparation of the Naph-PEG/PBA- $\gamma$-CyD PPRX

The synthesized mono modified PBA- $\gamma$-CyD shows high solubility $(>100 \mathrm{mM})$, similar to parent $\gamma$-CyD, which is suitable for the preparation of PPRXs. In contrast, PBA- $\alpha-C y D$ and PBA- $\beta-C y D$ shows low solubility because of the formation of supramolecular structures [36]. In a preliminary experiment, we used unmodified PEG to prepare a PPRX. PEG (MW 2000, $11.0 \mathrm{mg}, 5.50 \mu \mathrm{mol})$ and PBA- $\gamma-\mathrm{CyD}(100 \mathrm{mg}, 69.2 \mu \mathrm{mol})$ were dissolved in water $(500 \mu \mathrm{L})$, and the solution was kept at room temperature, but there was no precipitate. Then, the solution was kept at $60^{\circ} \mathrm{C}$. After 28 days, the resulting precipitate of the PPRX was filtered and dried under reduced pressure (55.0 mg). We considered that temperature of $60^{\circ} \mathrm{C}$ is too high to apply to protein drugs and realized that the modification of the terminal of PEG was effective to form PPRXs [12]. We modified both of the terminal hydroxyl groups of PEG with naphthoyl groups (Naph-PEG) and used the latter to prepare a PPRX. Naph-PEG (12.3 mg, $5.50 \mu \mathrm{mol})$ and PBA- $\gamma$-CyD (100 mg, 69.2 $\mu \mathrm{mol})$ were dissolved in water $(500 \mu \mathrm{L})$, and the solution was kept at room temperature. After 28 days, the resulting precipitates, which we considered to be a PPRX, were filtered and dried under reduced pressure (84.7 mg).

Structural analysis of the Naph-PEG/PBA- $\gamma$-CyD PPRX

To clarify whether or not PBA- $\gamma$-CyD includes Naph-PEG in the resulting precipitate, we measured it with DSC (Fig. 3) and XRD (Fig. 4). In the DSC thermograms of Naph-PEG, an endothermic peak was observed at $39^{\circ} \mathrm{C}$, corresponding the melting point of Naph-PEG. In the DSC thermograms of the Naph-PEG/PBA- $\gamma$-CD PPRX, the endothermic peak disappeared completely, which demonstrates that Naph-PEG is fully included in the cavities of $\gamma$-CyD [21].

The XRD pattern of the Naph-PEG/PBA- $\gamma$-CyD PPRX was highly crystalline, which supports the formation of PPRXs. However, the pattern was quite different from that of PPRXs using parent $\gamma$-CyD [20], suggesting that the obtained precipitate is a new type of PPRX. To investigate the stoichiometry, we dissolved the Naph-PEG/PBA- $\gamma$-CyD PPRX in DMSO- $d_{6}$ and measured results using ${ }^{1} \mathrm{H}$ NMR. The spectra showed that the stoichiometry between the ethylene glycol monomer unit and PBA- $\gamma-\mathrm{CyD}$ was $2: 1$. This value is similar to that of a PEG/ $\alpha-\mathrm{CyD}$ PPRX but not to that of a PEG/ $\gamma$-CyD PPRX. The PEG/ $\alpha-C y D$ PPRX is single stranded, and the stoichiometry between its ethylene glycol monomer unit and PBA- $\gamma-C y D$ is 2:1 [11]. In contrast, the PEG/ $\gamma$-CyD PPRX is double stranded, and its stoichiometry between the ethylene glycol monomer unit and PBA- $\gamma-C y D$ is 4:1 [12]. This result implies the formation of a single-stranded PPRX composed of Naph-PEG and PBA- $\gamma$-CyD. 
Fluorescence spectroscopy of the naphthoyl group at the terminal PEG chain provides information about whether the PPRX is single stranded or double stranded [12]. We monitored the solid-state fluorescence spectra of the Naph-PEG/PBA- $\gamma$-CyD PPRX and compared these spectra with those of the PPRX composed of Naph-PEG and parent CyDs (Fig. 5). The spectrum of Naph-PEG/ $\gamma$-CyD shows an excimer emission that is widened and shifted to a longer wavelength, which means that two naphthoyl groups were included within the cavity of the parent $\gamma$-CyD. This appearance of excimer emission proves that the Naph-PEG/ $\gamma$-CyD PPRX has double-stranded PEG chains. In contrast, the spectrum of the Naph-PEG/PBA- $\gamma$-CyD PPRX shows a peak at $363 \mathrm{~nm}$ derived from a monomeric naphthoyl group, indicating the formation of a single-stranded PPRX like the Naph-PEG/ $\alpha$-CyD PPRX. From the results of ${ }^{1} \mathrm{H}$ NMR and solid-state fluorescence spectroscopy, we conclude that the Naph-PEG/PBA- $\gamma$-CyD PPRX is single stranded. The formation of single-stranded PPRX with a guest-modified $\gamma$-CyD has never been reported before.

To gain an insight into the intermolecular interactions between building blocks, we measured the NMR spectra of PBA- $\gamma$-CyD in the dissolved state. The two-dimensional nuclear Overhauser effect spectroscopy (NOESY) spectrum of a solution containing PBA- $\gamma-\mathrm{CyD}(5.0 \mathrm{mM})$ in $\mathrm{D}_{2} \mathrm{O}$ showed the nuclear Overhauser effect (NOE) interaction between the protons of the phenyl group and the interior protons of the CyD's cavity (H-3 and H-5, Fig. S10), indicating that the PBA moiety was included by the CyD moiety. The one-dimensional ${ }^{1} \mathrm{H}$ NMR spectra of PBA- $\gamma-\mathrm{CyD}$ in $\mathrm{D}_{2} \mathrm{O}$ showed that the protons of the phenyl ring shifted as the concentration of PBA- $\gamma$-CyD increased (Fig. S11), indicating that the interaction of PBA and CyD ring is intermolecular but not intramolecular [37].

To investigate the spatial arrangement of Naph-PEG, we measured the rotating Overhauser enhancement and exchange spectroscopy (ROESY) spectrum of a solution containing Naph-PEG (0.4 mM) and PBA- $\gamma$-CyD (2.0 mM) in $\mathrm{D}_{2} \mathrm{O}$. In the spectrum, the NOE interaction between the protons of the phenyl group and the protons of the PEG moiety (Fig. S12) demonstrate that the PBA moiety and the PEG chain are present nearby.

To summarize these NMR results, a single-stranded PEG is included by the $\gamma$-CyD ring, and the remaining space is occupied by the PBA moiety because the single PEG chain is not sufficiently large to occupy the large cavity of $\gamma$-CyD.

Sugar response of the Naph-PEG/PBA- $\gamma$-CyD PPRX

We evaluated the sugar responses of the Naph-PEG/PBA- $\gamma$-CyD PPRX with respect to the solubility changes using turbidity measurement (Fig. 6) and change in the release rate of Naph-PEG by monitoring the fluorescence of terminal naphthoyl groups of PEG chains (Fig. 7). Naph-PEG/PBA- $\gamma$-CyD (6.0 mg) was suspended in a buffer solution (2.0 mL, $20 \mathrm{mM}$ HEPES buffer $\mathrm{pH} 7.4,37^{\circ} \mathrm{C}$ ). After the turbidity became constant, stock sugar solution was added to the suspended solution. The turbidity was decreased as the concentration of sugar increased, which suggests that the solid structure of the PPRX disintegrates and dissolves because of the effect of sugar. The effect of D-fructose (Fru) was larger than that of Glc, proving that the PBA moiety acts as a sugar-recognition moiety because PBA reacts more strongly with Fru than with Glc [27-30].

Fig. 7 shows the release rate of Naph-PEG in various conditions. The sugar addition accelerated the rate, which depends on the kind and concentration of sugars. These results show that we have successfully introduced sugar responsiveness into the PPRX for the first time; however, it is not sufficient for controlled release of Ins. About $10 \%$ of Naph-PEG was released after $8 \mathrm{~h}$, indicating that the PPRX is disintegrated in water by dilution. Although an ideal 
system has a clear on/off response for sugar, the current system shows a slow release of Naph-PEG in the absence of sugar. The PPRX should be more rigid in the absence of sugar. The structure of building blocks, polymer and modified $\mathrm{CyD}$, and their combination can affect the rigidity of the PPRX. At the same time, the PPRX should have a transformability that would be influenced by affinities of the PBA moiety for Glc and by the spatial arrangement of building blocks. The balance between rigidity and transformability are important and will be the focus of our future work.

Preparation of Naph-PEG-Ins/PBA- $\gamma$-CyD PPRX

We prepared Naph-PEG-Ins using heterobifunctional PEG $\left[\mathrm{H}_{2} \mathrm{~N}-\left(\mathrm{CH}_{2}\right)_{3}-\mathrm{O}-\mathrm{PEG}-\left(\mathrm{CH}_{2}\right)_{5}-\mathrm{COOH}\right.$. The amine end was modified with a naphthoyl group, and the carboxylic terminal was linked to amino groups of Ins (Fig. 1). The average ratio between PEG and Ins was calculated to be 2.2 from ${ }^{1} \mathrm{H}$ NMR spectra of Naph-PEG-Ins (Figs. S4 and S5). A solution of Naph-PEG-Ins was added to a solution of PBA- $\gamma$-CyD, and a Naph-PEG-Ins/PBA- $\gamma$-CyD PPRX was formed as a crystalline precipitate (Fig. S8). The results of ${ }^{1} \mathrm{H}$ NMR (Fig. S9), DSC (Fig. S13), and XRD (Fig. S14) were similar to those of the Naph-PEG/PBA- $\gamma$-CyD PPRX, indicating the Naph-PEG-Ins/PBA- $\gamma-C y D$ PPRX was successfully obtained.

Sugar response of the Naph-PEG-Ins/PBA- $\gamma$-CyD PPRX

We investigated the effect of sugar on the release of Naph-PEG-Ins from the Naph-PEG-Ins/PBA- $\gamma$-CyD PPRX. The suspension of Naph-PEG-Ins/PBA- $\gamma$-CyD PPRX was kept at $37^{\circ} \mathrm{C}$, and the amount of Naph-PEG-Ins dissolved into supernatant was evaluated by monitoring the fluorescence of the terminal naphthoyl group of Naph-PEG-Ins. Fig. 8 shows that the release rate is accelerated in the presence of sugar, and the release rate depends on the kind and concentrations of sugars.

Current guidelines recommend a post-meal Glc level of $<10 \mathrm{mM}$ and a fasting plasma Glc level of 3.9-7.2 mM [38]. Compared with the blood Glc level, the PPRX system requires a higher concentration of Glc. Our next challenge is to increase the Glc affinity of the PBA moiety. The PBA derivatives employed here are the simplest structures and showed a relatively low affinity for Glc. At present, there are many PBA-based Glc sensors that show a high affinity for Glc [3941]. For example, a fluorescent sugar sensor containing bis-boronic acid shows an elevated affinity that includes a clinically meaningful Glc level [41]. The introduction of a sophisticated bis-boronic acid derivative to the CyD ring perhaps would be a suitable system.

\section{Conclusion}

We have successfully developed a new model of a stimuli-responsive PPRX. The Naph-PEG/PBA- $\gamma$-CyD PPRX was insoluble in a $\mathrm{pH} 7.4$ solution; however, sugar addition induced disintegration of the PPRX. This model is applicable to PEG-Ins, and we demonstrated that the release rate of Naph-PEG-Ins was accelerated in the presence of sugar. It is worthy of attention that the Naph-PEG-Ins/PBA- $\gamma$-CyD PPRX works as a sugar responsive release system, in which Glc triggers the disintegration of the PPRX and the accompanying release of Naph-PEG-Ins. In other words, this is the first 
successful attempt to introduce a sugar responsiveness into PEG-Ins/CyD PPRXs that have been developed as a sustained release system for PEG-Ins [22-24].

In this study, we also discovered that PPRXs using PBA- $\gamma-C y D$ were single stranded despite the large cavity of $\gamma$-CyD. We demonstrated that the non-covalent molecular interaction between a PBA moiety and a CyD cavity is important to form a single-stranded PPRX. In further studies, we must increase the affinity of PBA derivatives for Glc. To address this issue, we will attempt to introduce various PBA derivatives into the $\gamma$-CyD ring.

\section{Acknowledgements}

This work was supported by JSPS KAKENHI Grant Number 25860027.

\section{References}

1. $\quad$ Fujisaki, Y., Tsukune, T., Funyû, M., Okumura, M., Ukigaya, T., Sugibayashi, K.: Development of sustained-release tablets containing sodium valproate: in vitro and in vivo correlation. Drug Dev. Ind. Pharm. 32, 207-217 (2006).

2. Juni, K., Nakano, M., Kubota, M.: Controlled release of aclarubicim, an anticancer antibiotic, from poly- $\beta$-hydroxybutyric acid microspheres. J. Control. Release. 4, 25-32 (1986).

3. Okada, H., Toguchi, H.: Biodegradable microspheres in drug delivery. Crit. Rev. Ther. Drug Carr. Syst. 12, 1-99 (1995).

4. Seki, T., Kawaguchi, T., Juni, K., Sugibayashi, K., Morimoto, Y.: Sustained transdermal delivery of zidovudine via controlled release of penetration enhancer. J. Control. Release. 17, 41-47 (1991).

5. Muijsers, R.B.R., Wagstaff, A.J.: Transdermal fentanyl: an updated review of its pharmacological properties and therapeutic efficacy in chronic cancer pain control. Drugs. 61, 2289-2307 (2001).

6. Qiu, Y., Park, K.: Environment-sensitive hydrogels for drug delivery. Adv. Drug Deliv. Rev. 53, 321-339 (2001).

7. Meng, F., Zhong, Z., Feijen, J.: Stimuli-responsive polymersomes for programmed drug delivery.

Biomacromolecules. 10, 197-209 (2009).

8. Sato, K., Takahashi, S., Anzai, J.: Layer-by-layer thin films and microcapsules for biosensors and controlled release. Anal. Sci. 28, 929-938 (2012).

9. Harada, A.: Cyclodextrin-based molecular machines. Acc. Chem. Res. 34, 456-464 (2001).

10. Liu, P., Cai, W., Chipot, C., Shao, X.: Thermodynamic insights into the dynamic switching of a cyclodextrin in a bistable molecular shuttle. J. Phys. Chem. Lett. 1, 1776-1780 (2010). 
11. Harada, A., Li, J., Kamachi, M.: The molecular necklace: a rotaxane containing many threaded $\alpha$-cyclodextrins. Nature. 356, 325-327 (1992).

12. Harada, A., Li, J., Kamachi, M.: Double-stranded inclusion complexes of cyclodextrin threaded on poly (ethylene glycol). Nature. 370, 126-128 (1994).

13. Harada, A., Takashima, Y., Yamaguchi, H.: Cyclodextrin-based supramolecular polymers. Chem. Soc. Rev. 38, 875-882 (2009).

14. Ooya, T., Choi, H.S., Yamashita, A., Yui, N., Sugaya, Y., Kano, A., Maruyama, A., Akita, H., Ito, R., Kogure, K., Harashima, H.: Biocleavable polyrotaxane-plasmid DNA polyplex for enhanced gene delivery. J. Am. Chem. Soc. 128, 3852-3853 (2006).

15. Park, C., Oh, K., Lee, S.C., Kim, C.: Controlled release of guest molecules from mesoporous silica particles based on a pH-responsive polypseudorotaxane motif. Angew. Chem. Int. Ed. Engl. 46, 1455-1457 (2007).

16. Li, J., Loh, X.J.: Cyclodextrin-based supramolecular architectures: syntheses, structures, and applications for drug and gene delivery. Adv. Drug Deliv. Rev. 60, 1000-1017 (2008).

17. Li, J.J., Zhao, F., Li, J.: Polyrotaxanes for applications in life science and biotechnology. Appl. Microbiol. Biotechnol. 90, 427-443 (2011).

18. Seo, J.-H., Kakinoki, S., Inoue, Y., Yamaoka, T., Ishihara, K., Yui, N.: Inducing rapid cellular response on RGD-binding threaded macromolecular surfaces. J. Am. Chem. Soc. 135, 5513-5516 (2013).

19. Ohya, Y., Takamido, S., Nagahama, K., Ouchi, T., Katoono, R., Yui, N.: Polyrotaxane composed of poly-L-lactide and alpha-cyclodextrin exhibiting protease-triggered hydrolysis. Biomacromolecules. 10, 22612267 (2009).

20. Higashi, T., Hirayama, F., Yamashita, S., Misumi, S., Arima, H., Uekama, K.: Slow-release system of pegylated lysozyme utilizing formation of polypseudorotaxanes with cyclodextrins. Int. J. Pharm. 374, 26-32 (2009).

21. Abu Hashim, I.I., Higashi, T., Anno, T., Motoyama, K., Abd-elgawad, A.-E.H., El-shabouri, M.H., Borg, T.M., Arima, H.: Potential use of $\gamma$-cyclodextrin polypseudorotaxane hydrogels as an injectable sustained release system for insulin. Int. J. Pharm. 392, 83-91 (2010).

22. Higashi, T., Hirayama, F., Arima, H., Uekama, K.: Polypseudorotaxanes of pegylated insulin with cyclodextrins: application to sustained release system. Bioorg. Med. Chem. Lett. 17, 1871-1874 (2007).

23. Higashi, T., Hirayama, F., Misumi, S., Arima, H., Uekama, K.: Design and evaluation of polypseudorotaxanes of pegylated insulin with cyclodextrins as sustained release system. Biomaterials. 29, 3866-3871 (2008). 
24. Higashi, T., Hirayama, F., Misumi, S., Motoyama, K., Arima, H., Uekama, K.: Polypseudorotaxane formation of randomly-pegylated insulin with cyclodextrins: slow release and resistance to enzymatic degradation. Chem. Pharm. Bull. 57, 541-544 (2009).

25. Veronese, F.M.: Peptide and protein PEGylation: a review of problems and solutions. Biomaterials. 22, 405-417 (2001).

26. Roberts, M.J., Bentley, M.D., Harris, J.M.: Chemistry for peptide and protein PEGylation. Adv. Drug Deliv. Rev. 54, 459-476 (2002).

27. James, T.D., Sandanayake, K.R.A.S., Shinkai, S.: Saccharide sensing with molecular receptors based on boronic acid. Angew. Chem., Int. Ed. Engl. 35, 1910-1922 (1996).

28. Egawa, Y., Seki, T., Takahashi, S., Anzai, J.: Electrochemical and optical sugar sensors based on phenylboronic acid and its derivatives. Mater. Sci. Eng. C. 31, 1257-1264 (2011).

29. Fossey, J.S., D’Hooge, F., van den Elsen, J.M.H., Pereira Morais, M.P., Pascu, S.I., Bull, S.D., Marken, F., Jenkins, a T. a, Jiang, Y.-B., James, T.D.: The development of boronic acids as sensors and separation tools. Chem. Rec. 12, 464-478 (2012).

30. Egawa, Y., Miki, R., Seki, T.: Colorimetric sugar sensing using boronic acid-substituted azobenzenes. Materials (Basel). 7, 1201-1220 (2014).

31. Yamauchi, A., Sakashita, Y., Hirose, K., Hayashita, T., Suzuki, I.: Pseudorotaxane-type fluorescent receptor exhibiting unique response to saccharides. Chem. Commun. 4312-4314 (2006).

32. Ozawa, R., Hayashita, T., Matsui, T., Nakayama, C., Yamauchi, A., Suzuki, I.: Effects of cyclodextrins and saccharides on dual fluorescence of N,N-dimethyl-4-aminophenylboronic acid in water. J. Incl. Phenom. Macrocycl. Chem. 60, 253-261 (2007).

33. Hoeg-jensen, T., Havelund, S., Nielsen, P.K., Markussen, J.: Reversible insulin self-assembly under carbohydrate control. J. Am. Chem. Soc. 127, 6158-6159 (2005).

34. Matsumoto, A., Yoshida, R., Kataoka, K.: Glucose-responsive polymer gel bearing phenylborate derivative as a glucose-sensing moiety operating at the physiological pH. Biomacromolecules. 5, 1038-1045 (2004).

35. Kim, H., Kang, Y.J., Kang, S., Kim, K.T.: Monosaccharide-responsive release of insulin from polymersomes of polyboroxole block copolymers at neutral pH. J. Am. Chem. Soc. 134, 4030-4033 (2012).

36. Nakamura, K., Seki, T., Egawa, Y., Miki, R., Oda, Y., Yamanoi, T., Seki, T.: Sugar-sensitive supramolecular structures based on phenylboronic acid modified cyclodextrins. 61, 1188-1191 (2013). 
37. Harada, A., Miyauchi, M., Hoshino, T.: Supramolecular polymers formed by cinnamoyl cyclodextrins. J. Polym. Sci. A Polym. Chem. 41, 3519-3523 (2003).

38. American Diabetes Association: Standards of medical care in diabetes--2014. Diabetes Care. 37 Suppl 1, S14-80 (2014).

39. Fang, H., Kaur, G., Wang, B.: Progress in boronic acid-based fluorescent glucose sensors. J. Fluoresc. 14, 481489 (2004).

40. Yan, J., Fang, H., Wang, B.: Boronolectins and fluorescent boronolectins: an examination of the detailed chemistry issues important for the design. Med. Res. Rev. 25, 490-520 (2005).

41. James, T.D., Sandanayake, K.R.A.S., Iguchi, R., Shinkai, S.: Novel saccharide-photoinduced electron transfer sensors based on the interaction of boronic acid and amine. J. Am. Chem. Soc. 117, 8982-8987 (1995).

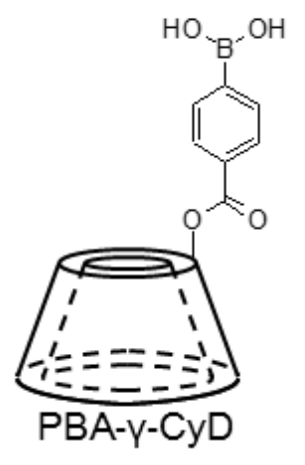

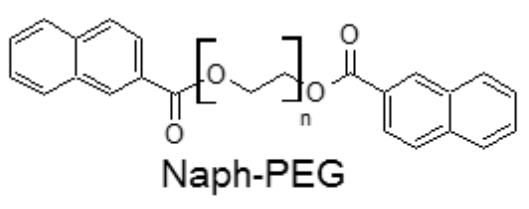

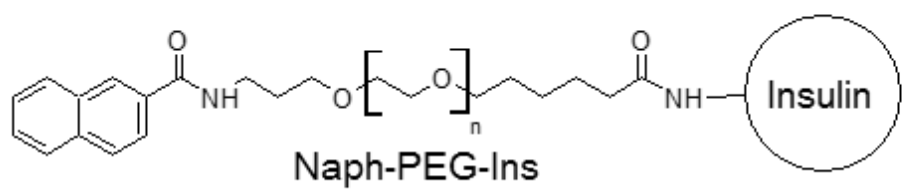

Fig. 1. Chemical structures of building blocks of sugar-responsive PPRXs. 


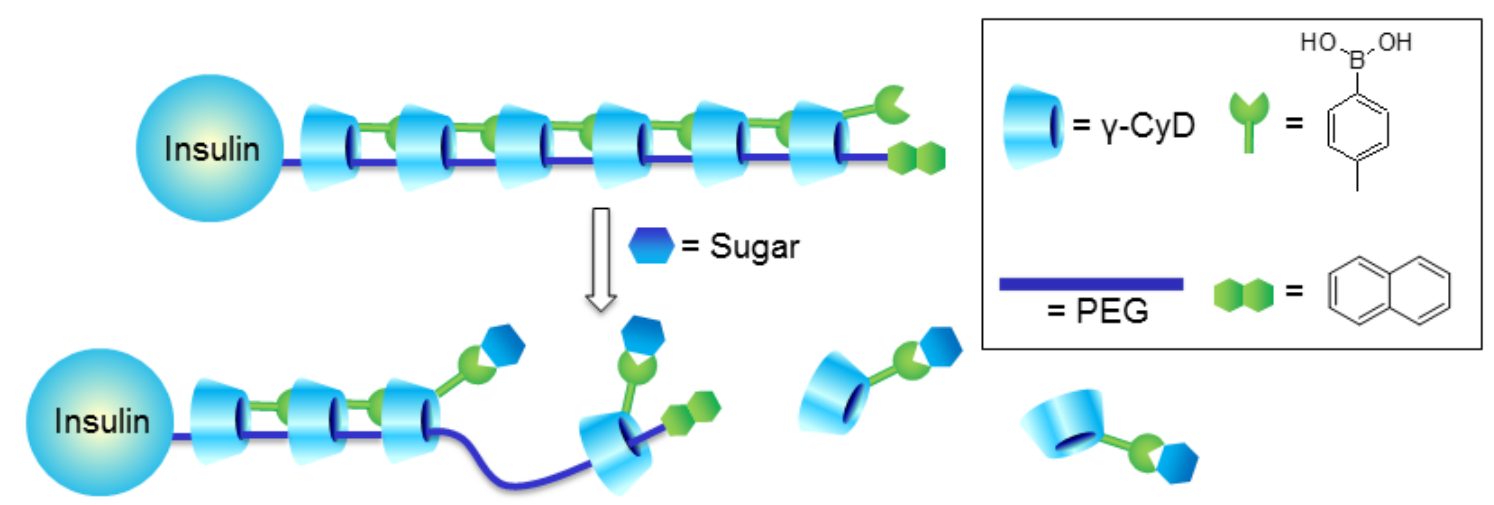

Fig. 2. Schematic illustration of sugar-induced disintegration of Naph-PEG-Ins/PBA- $\gamma-C y D$ PPRX.

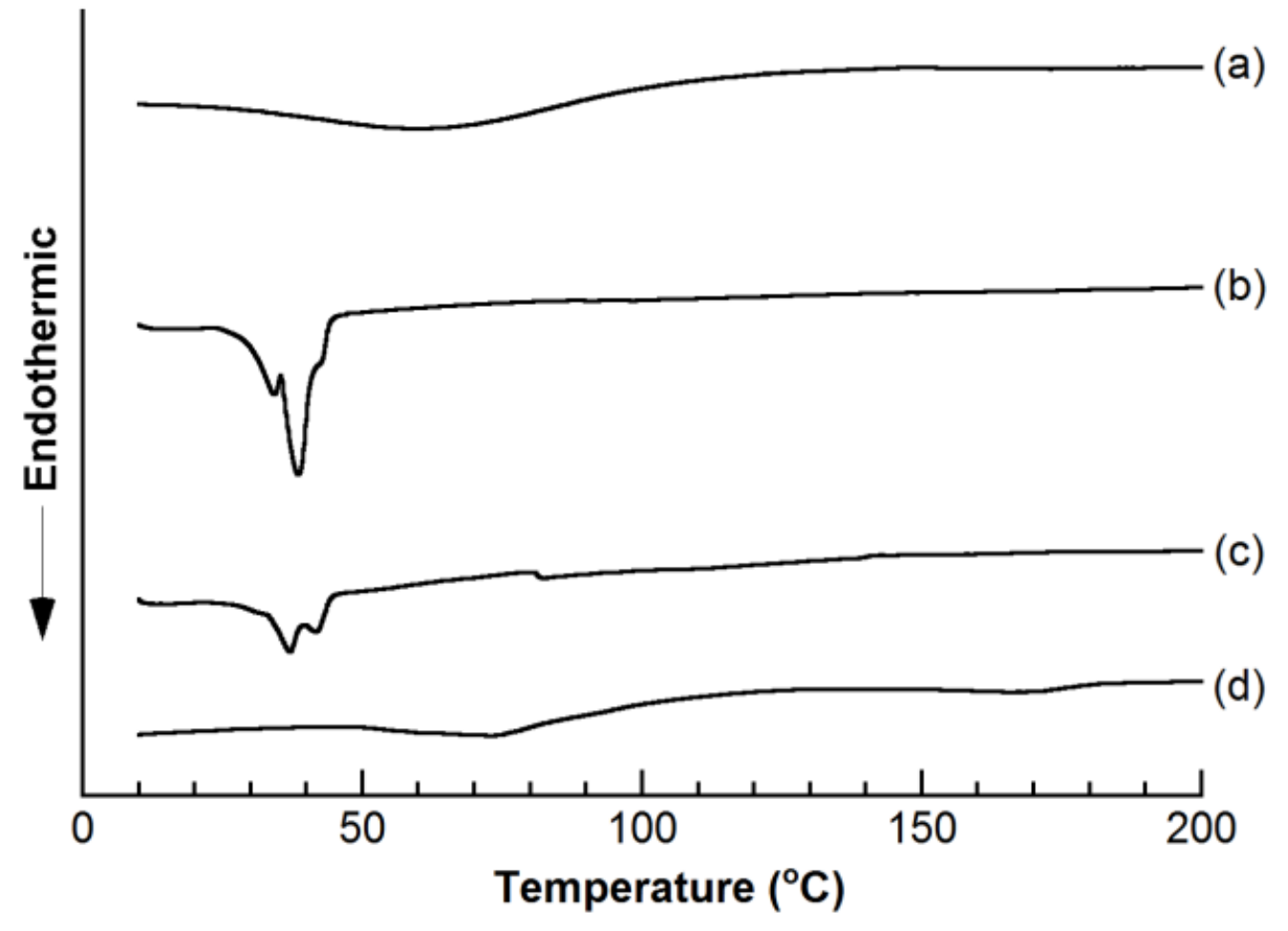

Fig. 3. DSC thermograms: (a) PBA- $\gamma-C y D$, (b) Naph-PEG, (c) physical mixture of Naph-PEG and PBA- $\gamma-C y D$, and (d) Naph-PEG/PBA- $\gamma$-CyD PPRX. 


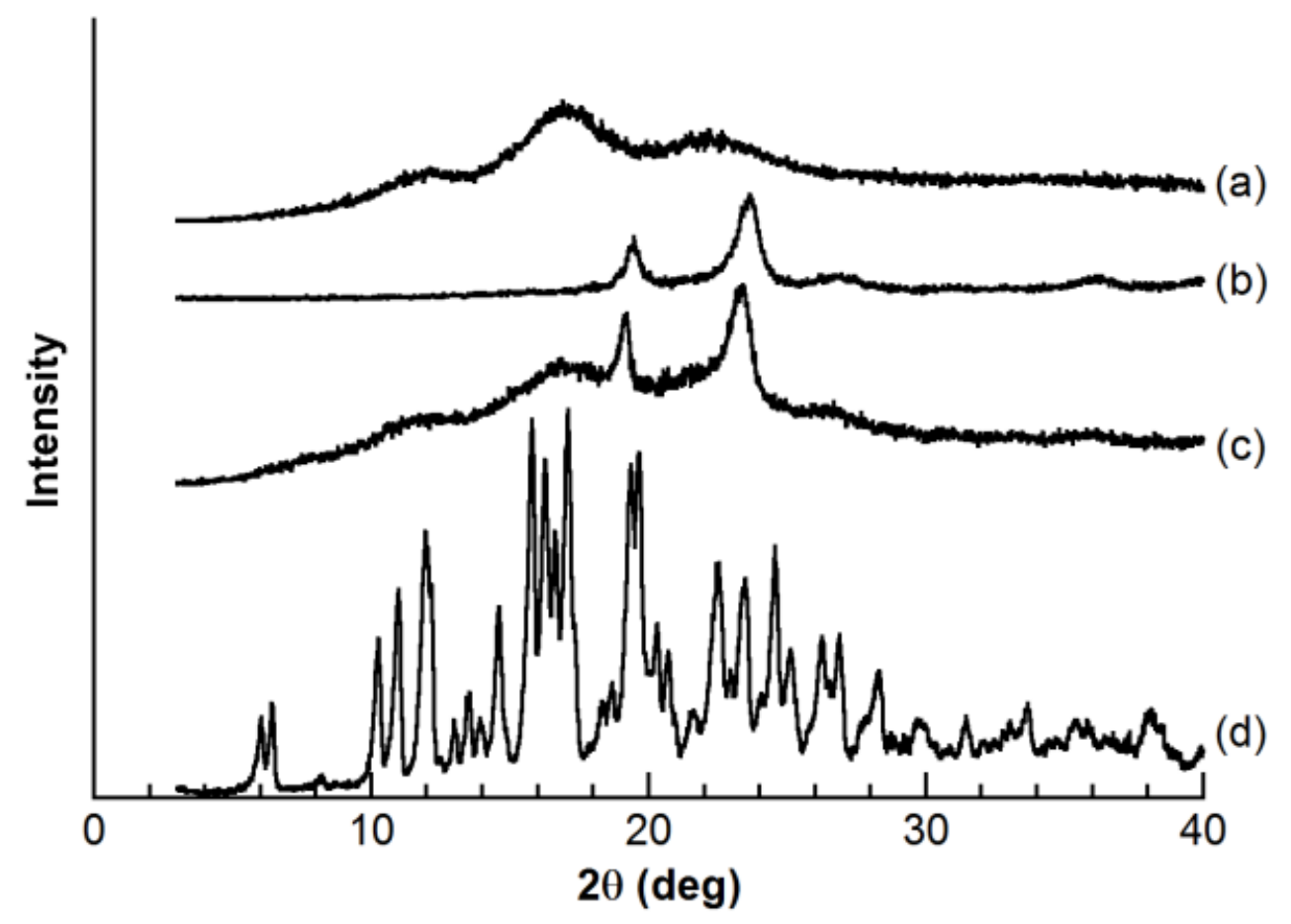

Fig. 4. XRD patterns: (a) PBA- $\gamma-C y D$, (b) Naph-PEG, (c) physical mixture of Naph-PEG and PBA- $\gamma-C y D$, and (d) Naph-PEG/PBA- $\gamma$-CyD PPRX.

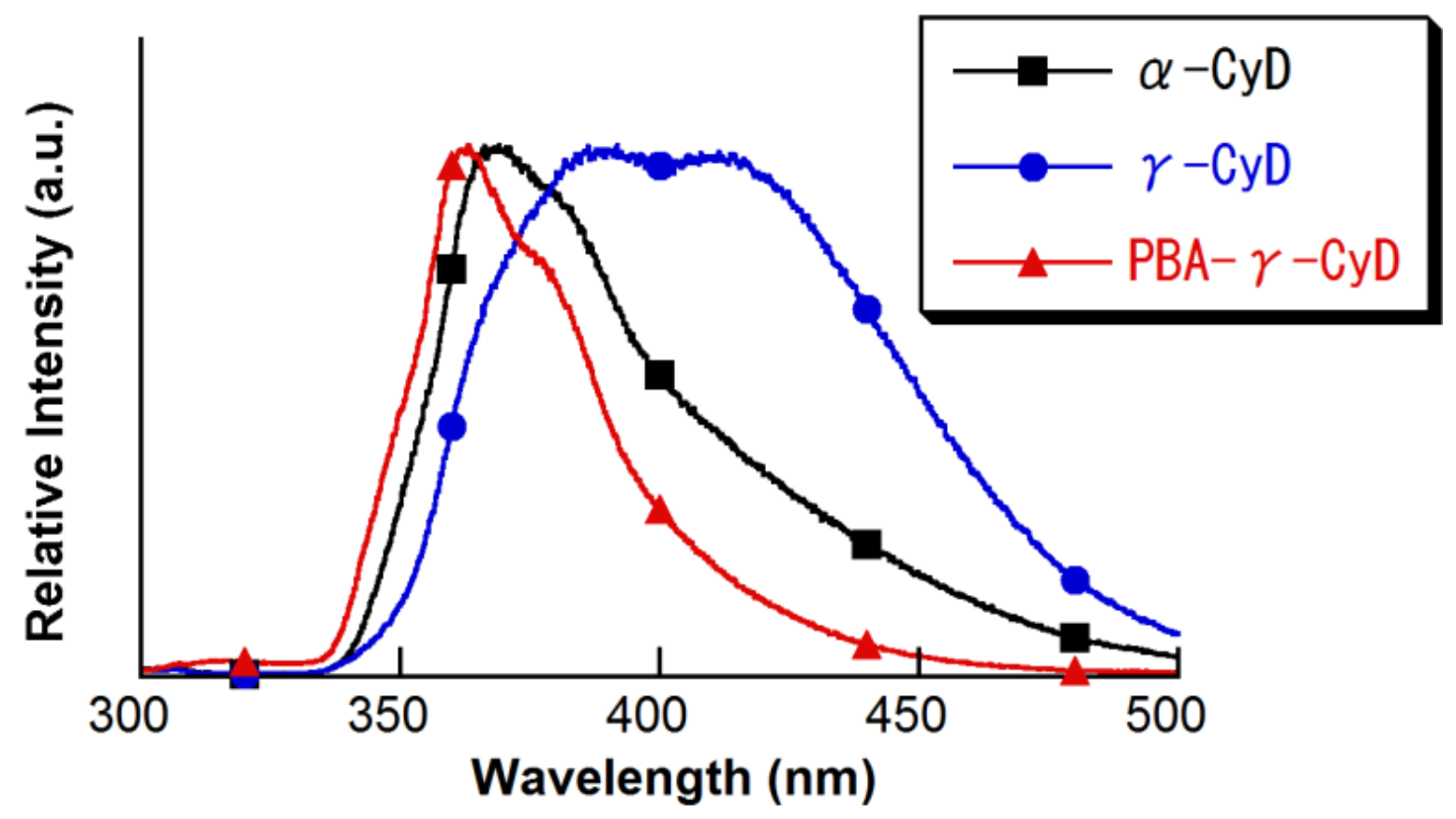

Fig. 5. Solid-state fluorescence spectra of Naph-PEG with $\alpha$-CyD, $\gamma$-CyD, or PBA- $\gamma$-CyD. 


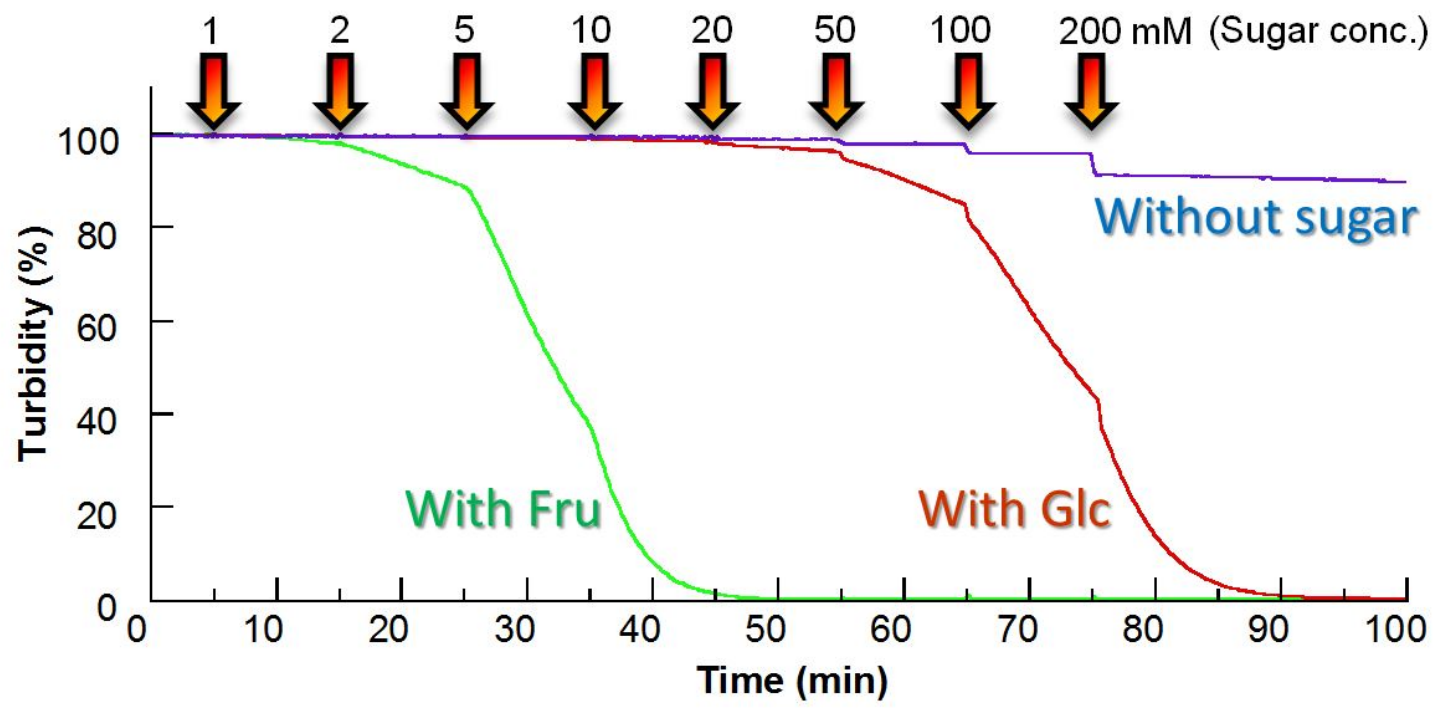

Fig. 6. The turbidity change of the Naph-PEG/PBA- $\gamma$-CyD PPRX depending on the sugar concentration $\left(\mathrm{pH} 7.4,37^{\circ} \mathrm{C}\right)$.

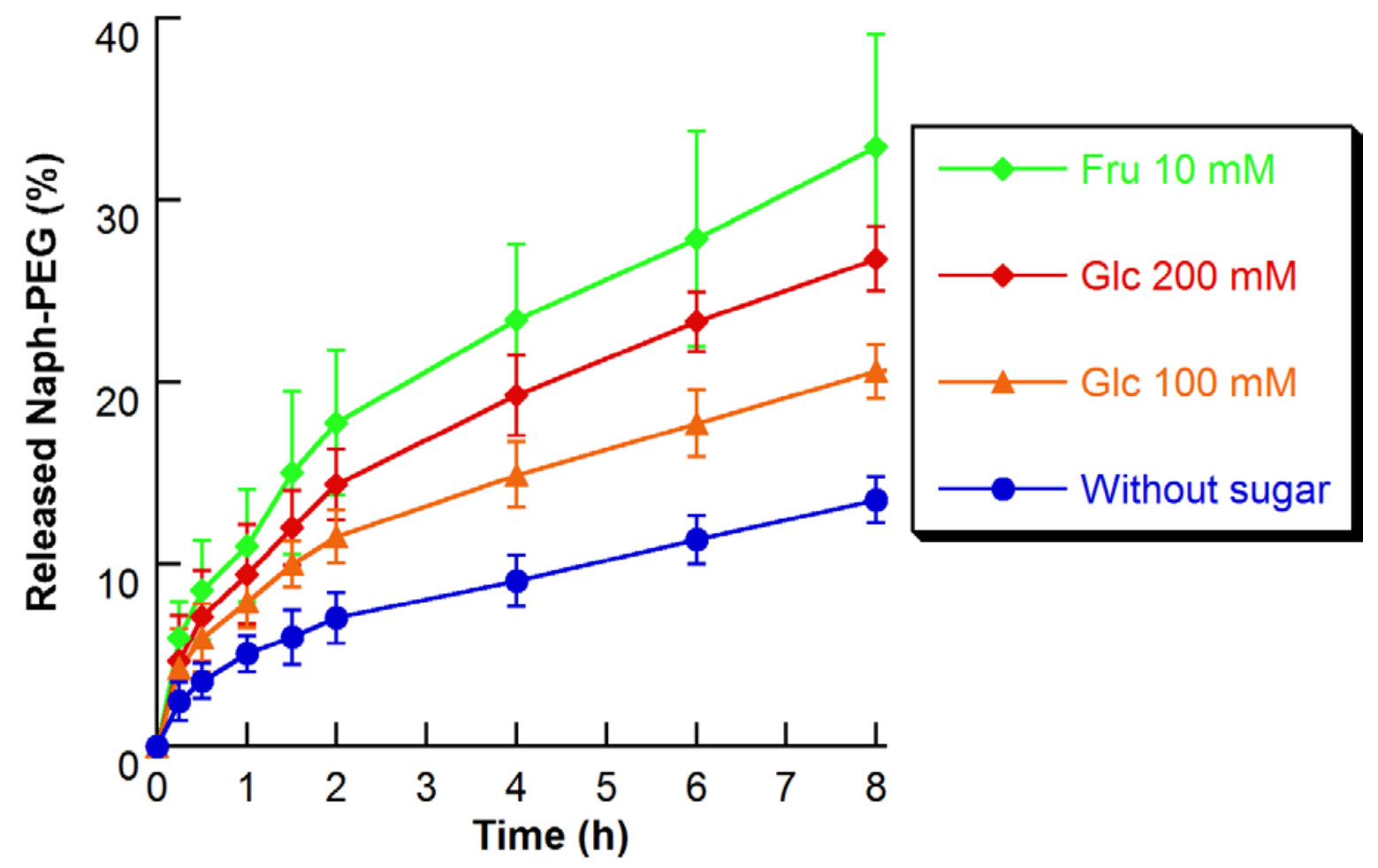

Fig. 7. Release profiles of Naph-PEG from the Naph-PEG/PBA- $\gamma$-CyD PPRX in the absence and presence of sugars (pH $\left.7.4,37^{\circ} \mathrm{C}\right)$. 


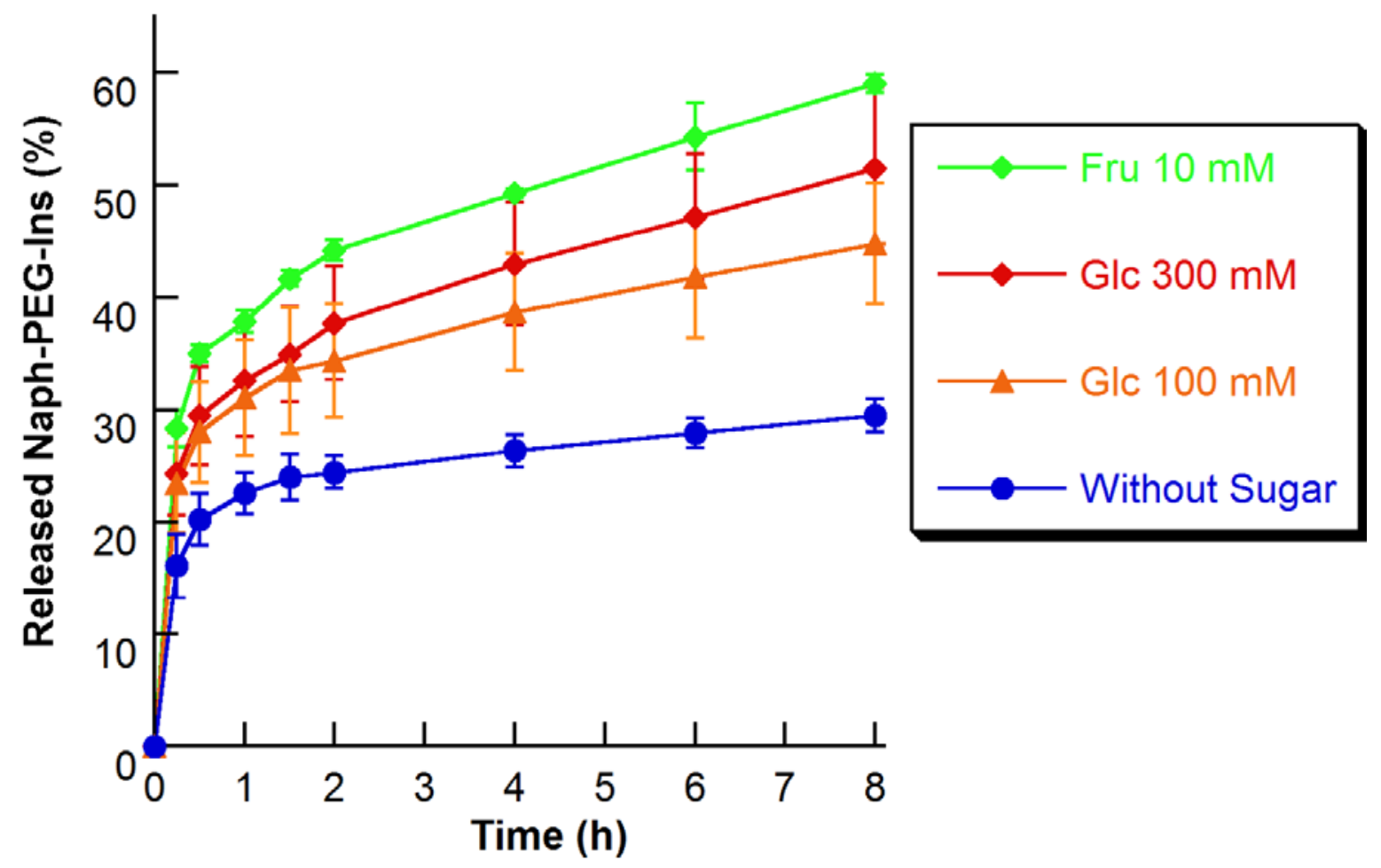

Fig. 8. Release profiles of Naph-PEG-Ins from the Naph-PEG-Ins/PBA- $\gamma$-CyD PPRX in the absence and presence of sugars $\left(\mathrm{pH} 7.4,37^{\circ} \mathrm{C}\right)$. 


\section{Supplementary Data}

\section{Sugar-responsive pseudopolyrotaxanes and their application in sugar-induced release of PEGylated insulin}

Tomohiro Seki, Keigo Abe, Kiminobu Nakamura, Yuya Egawa,* Ryotaro Miki, Kazuhiko Juni, Toshinobu Seki

Faculty of Pharmaceutical Sciences, Josai University, Keyakidai, Sakado, Saitama 350-0295, Japan.

Contents

1. NMR spectra and optical micrographs

(1) Phenylboronic acid modified $\gamma$-CyD (PBA- $\gamma$-CyD)

(2) Naphthalene-modified polyethylene glycol (Naph-PEG)

(3) One terminal naphthalene-modified polyethylene glycol (One-Naph-PEG)

(4) Naph-PEG-appended insulin (Naph-PEG-Ins)

(5) Naph-PEG/PBA- $\gamma-C y D$ PPRX

(6) Naph-PEG-Ins/PBA- $\gamma$-CyD PPRX

(7) NOESY spectrum of PBA- $\gamma$-CyD

(8) PBA- $\gamma$-CyD in various concentrations

(9) ROESY spectrum of PBA- $\gamma$-CyD with Naph-PEG

2. DSC thermograms of Naph-PEG-Ins/PBA- $\gamma$-CyD PPRX

3. XRD patterns of Naph-PEG-Ins/PBA- $\gamma-C y D$ PPRX 
1. NMR spectra

(1) Phenylboronic acid modified $\gamma$-CyD (PBA- $\gamma$-CyD)

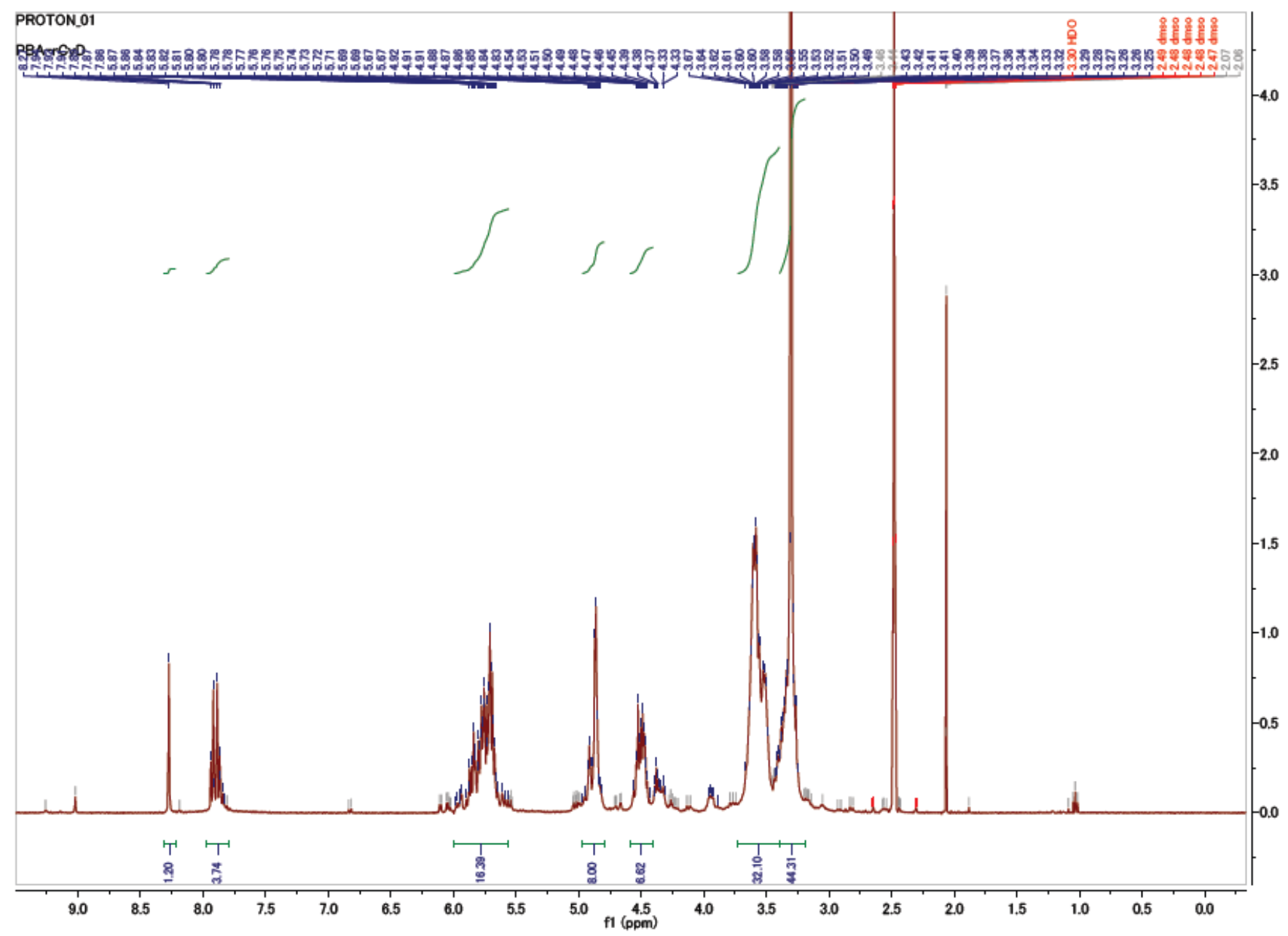

Fig. S1. The ${ }^{1} \mathrm{H}$ NMR spectrum of PBA- $\gamma-\mathrm{CyD}$ in DMSO- $d_{6}$ at $25^{\circ} \mathrm{C}$. 
(2) Naphthalene-modified polyethylene glycol (Naph-PEG)

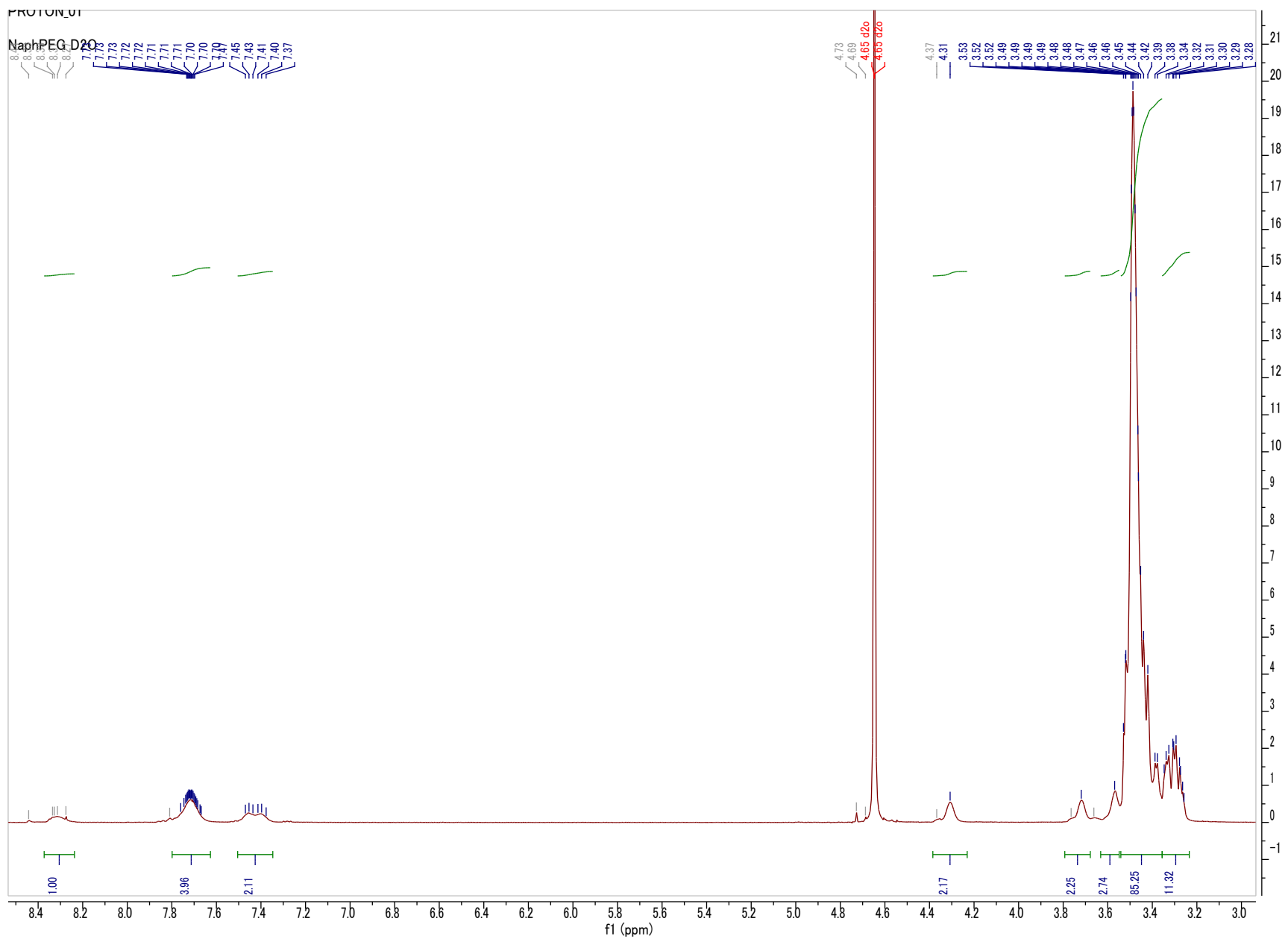

Fig. S2. The ${ }^{1} \mathrm{H}$ NMR spectrum of Naph-PEG in $\mathrm{D}_{2} \mathrm{O}$ at $25^{\circ} \mathrm{C}$. 
(3) One terminal naphthalene-modified polyethylene glycol (One-Naph-PEG)

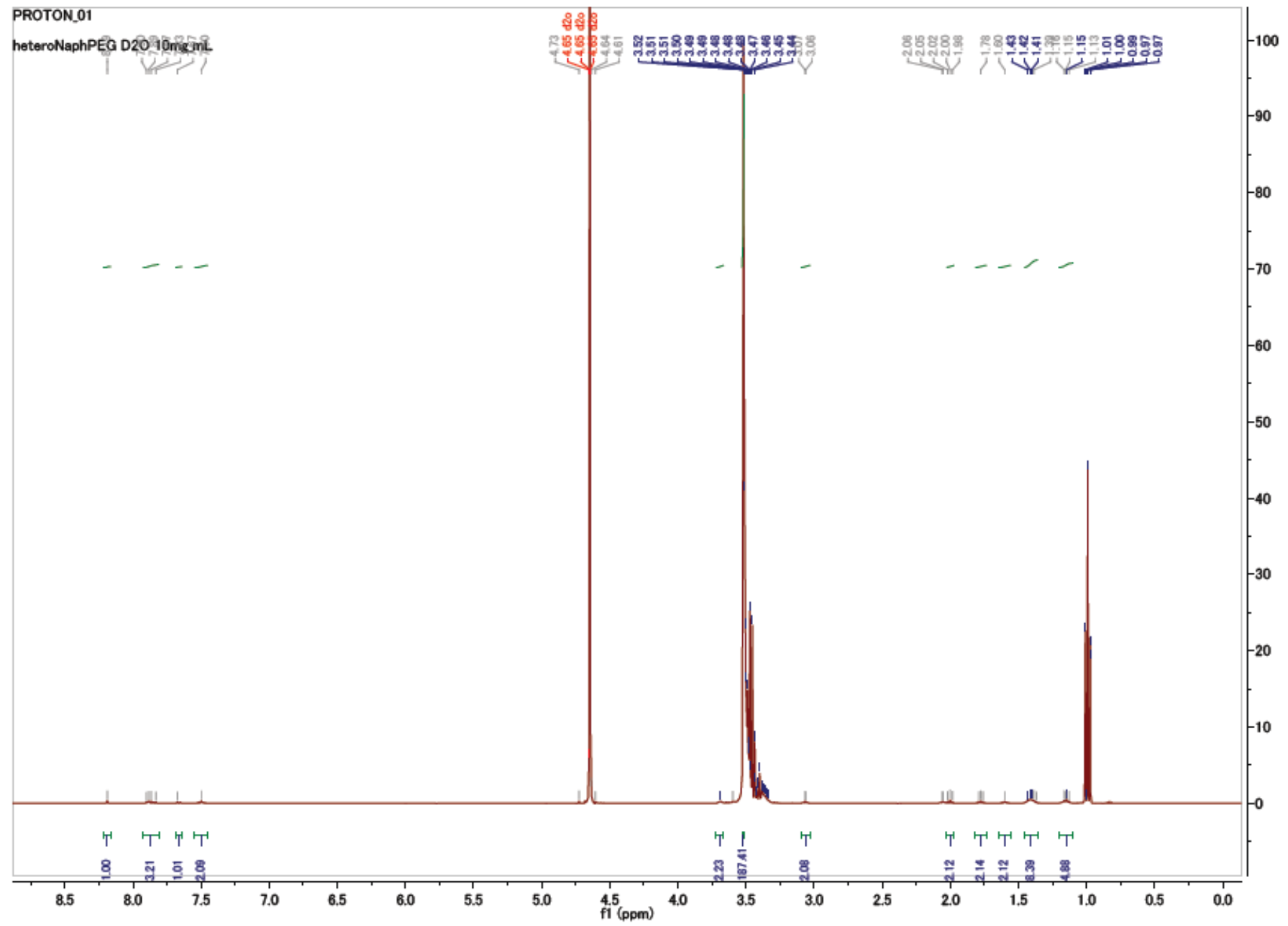

Fig. S3. The ${ }^{1} \mathrm{H}$ NMR spectrum of One-Naph-PEG in $\mathrm{D}_{2} \mathrm{O}$ at $25^{\circ} \mathrm{C}$. 
(4) Naph-PEG-appended insulin (Naph-PEG-Ins)

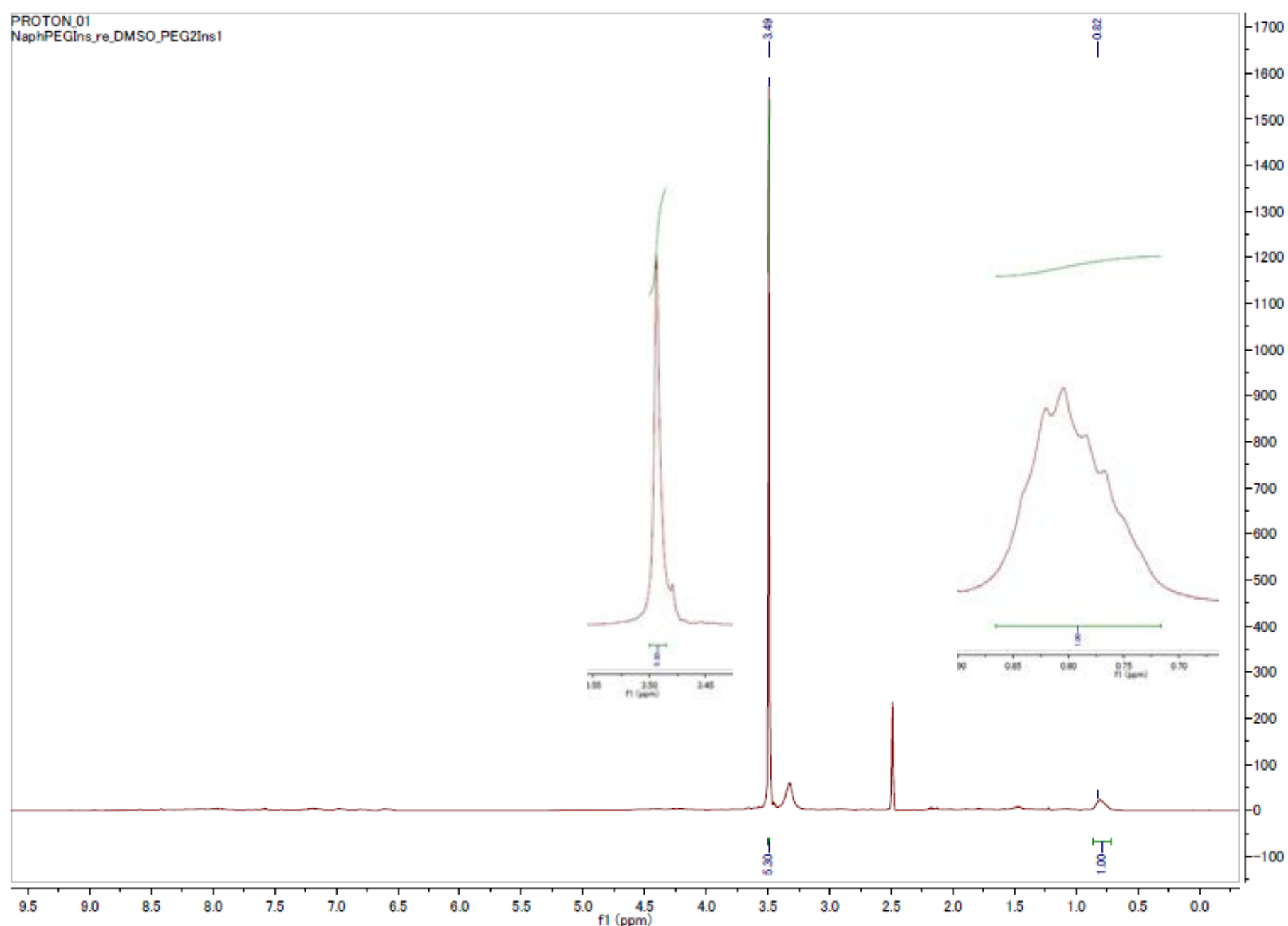

Fig. S4. The ${ }^{1} \mathrm{H}$ NMR spectrum of Naph-PEG-Ins in DMSO- $d_{6}$ at $25^{\circ} \mathrm{C}$, and the enlarged views of signals at 0.7-09 ppm and 3.5 ppm.

In order to evaluate the stoichiometry of PEG/Ins in the obtained Naph-PEG-Ins, DMSO- $d_{6}$ solutions containing PEG and Ins with various molar ratio $(0.0,1.0,1.5,3.0,6.0,12.0)$, and they were measured with ${ }^{1} \mathrm{H}$ NMR. The integrated intensity of the signals at 0.7-0.9 ppm derived from insulin and that at integration of the signal of PEG at 3.5 ppm was evaluated. The PEG/Ins intensity ratio was plotted against PEG/Ins molar ratio to obtain linear standard curve. When Naph-PEG-Ins was dissolved in DMSO- $d_{6}$ and monitored with ${ }^{1} \mathrm{H}$ NMR, the PEG/Ins intensity ratio was 5.5, which means the PEG/Ins molar ratio is 2.2 in Naph-PEG-Ins.

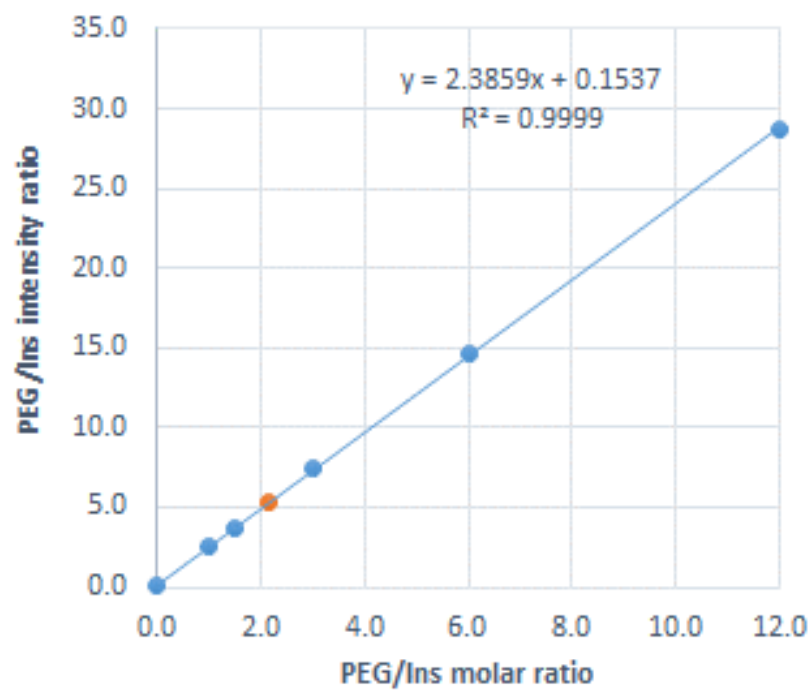

Fig. S5. The liner standard curve for PEG/Ins ratio. 
(5) Naph-PEG/PBA- $\gamma-C y D$ PPRX

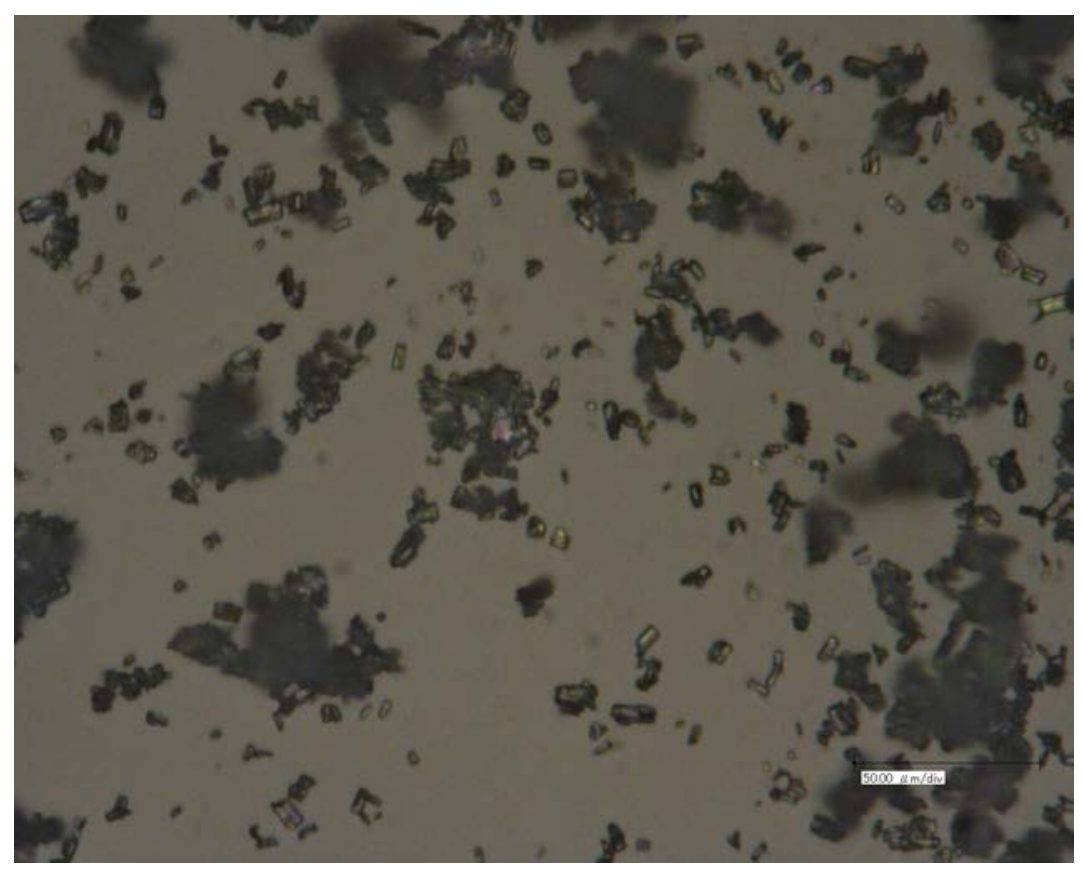

Fig. S6. An optical micrograph of Naph-PEG/PBA- $\gamma-C y D$ PPRX.

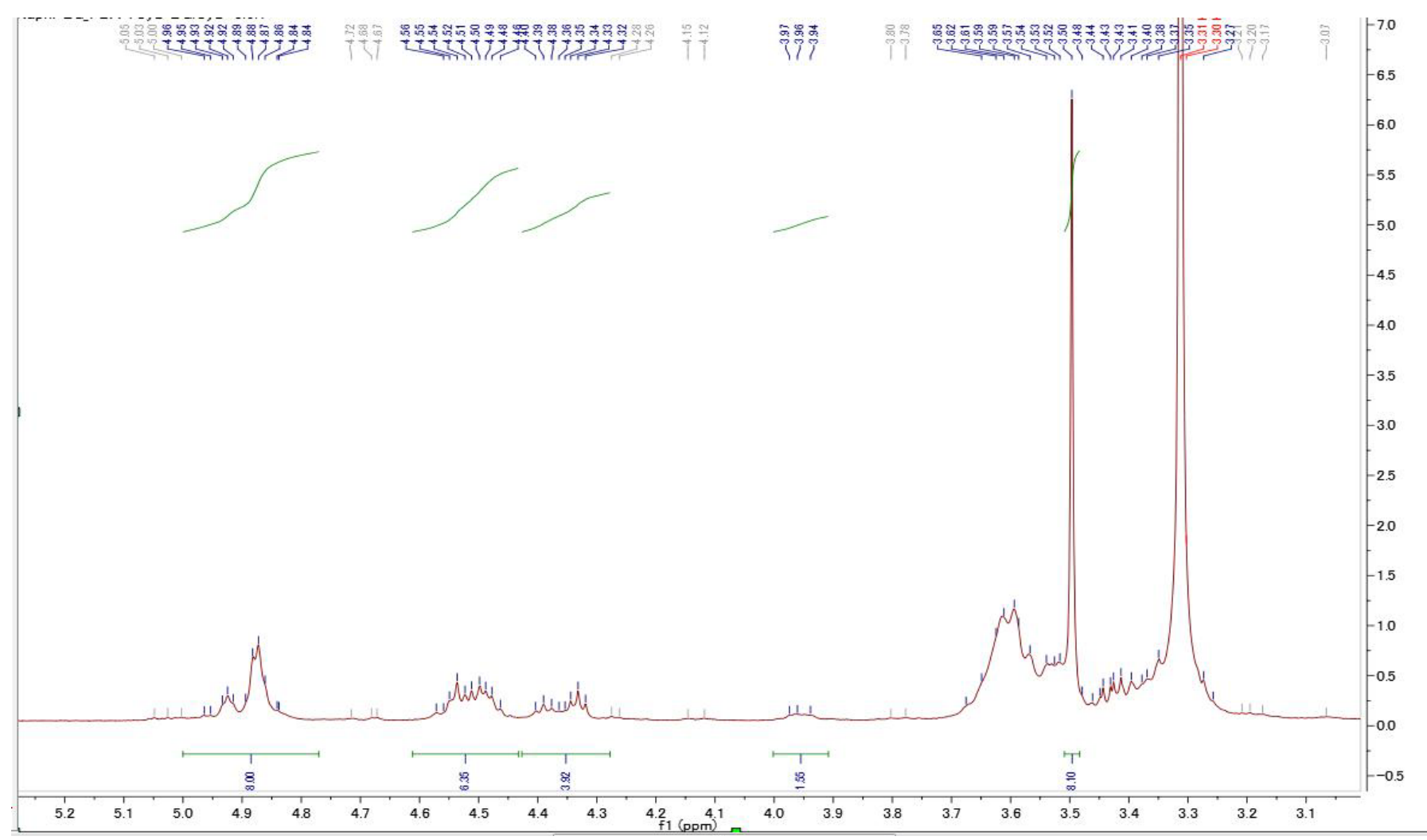

Fig. S7. The ${ }^{1} \mathrm{H}$ NMR spectrum of Naph-PEG/PBA- $\gamma-C y D$ PPRX in DMSO- $d_{6}$ at $25^{\circ} \mathrm{C}$. 
(6) Naph-PEG-Ins/PBA- $\gamma$-CyD PPRX

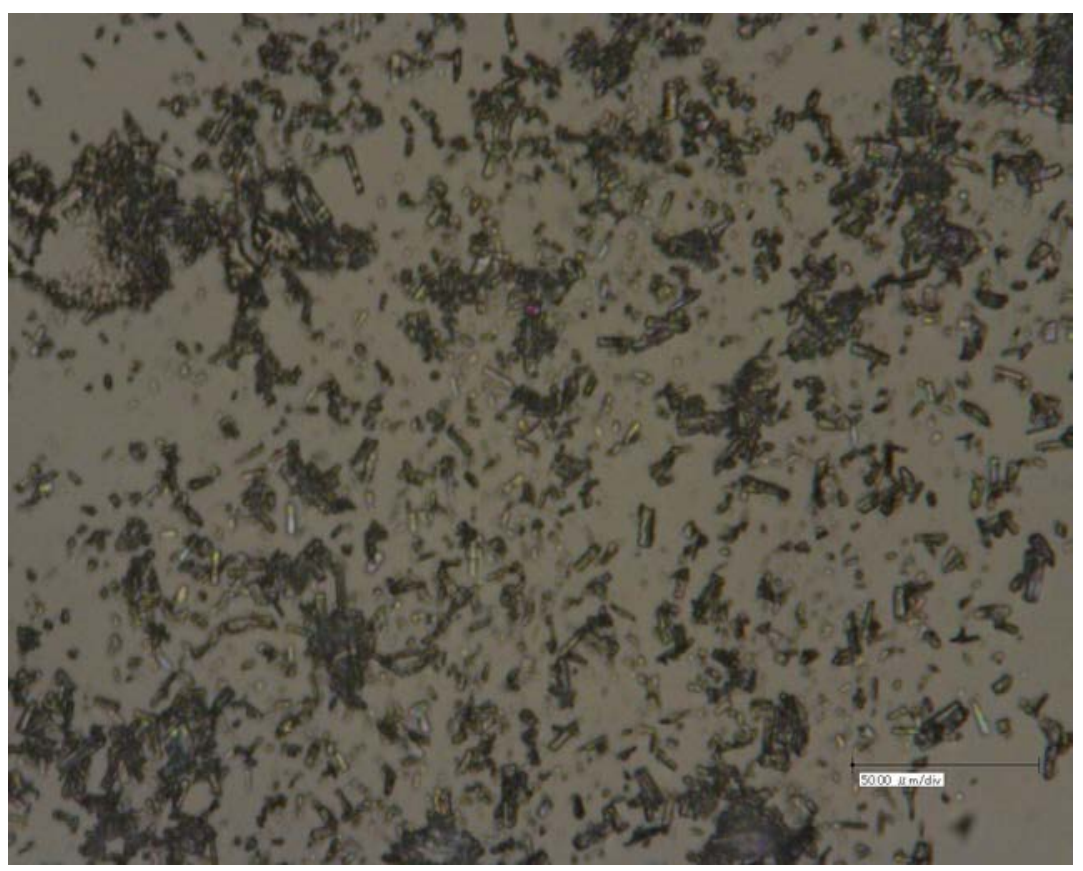

Fig. S8. An optical micrograph of Naph-PEG-Ins/PBA- $\gamma-C y D$ PPRX.

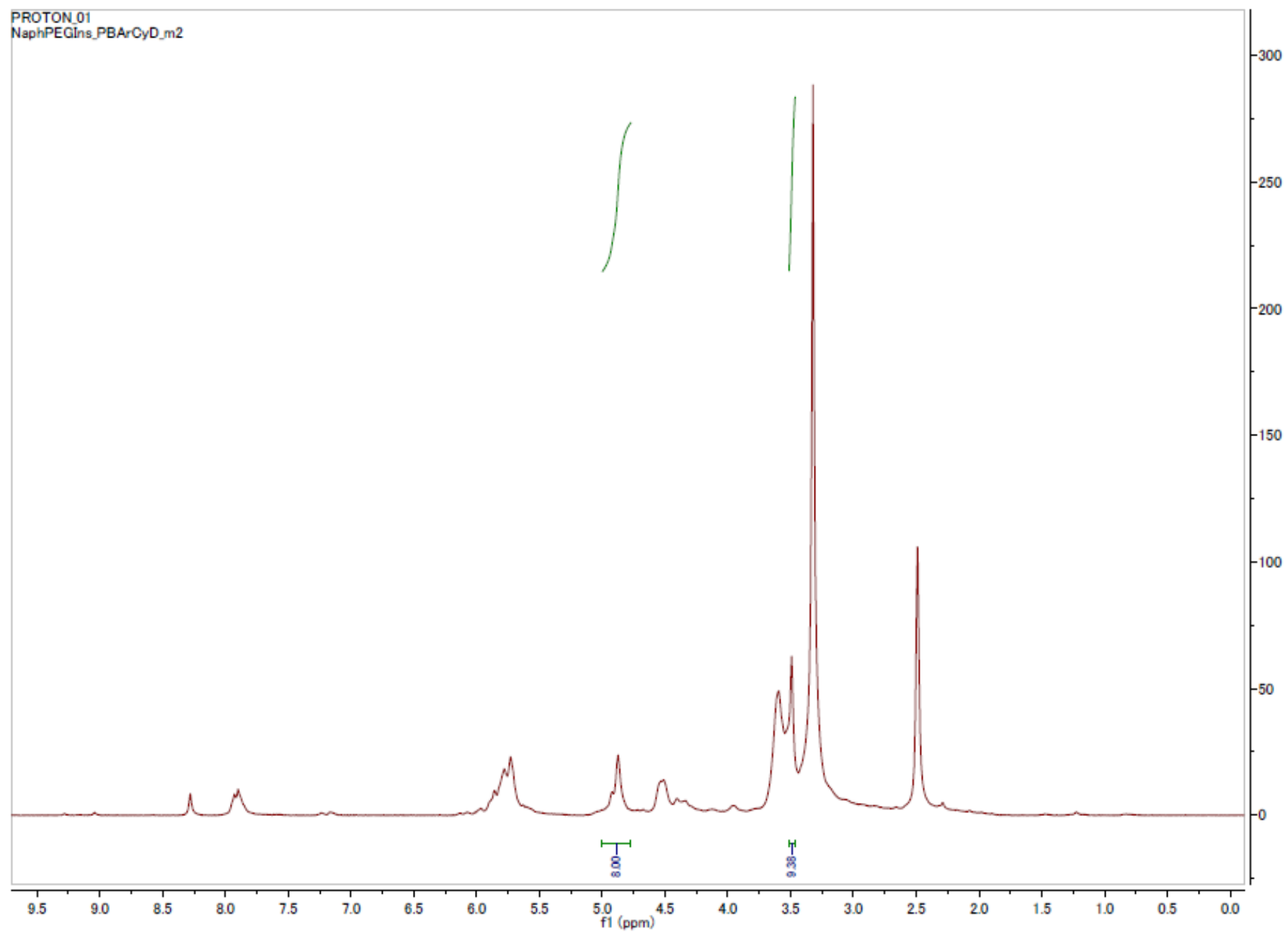

Fig. S9. The ${ }^{1} \mathrm{H}$ NMR spectrum of Naph-PEG-Ins/PBA- $\gamma$-CyD PPRX in DMSO- $d_{6}$ at $25^{\circ} \mathrm{C}$. 
(7) NOESY spectrum of PBA- $\gamma-C y D$
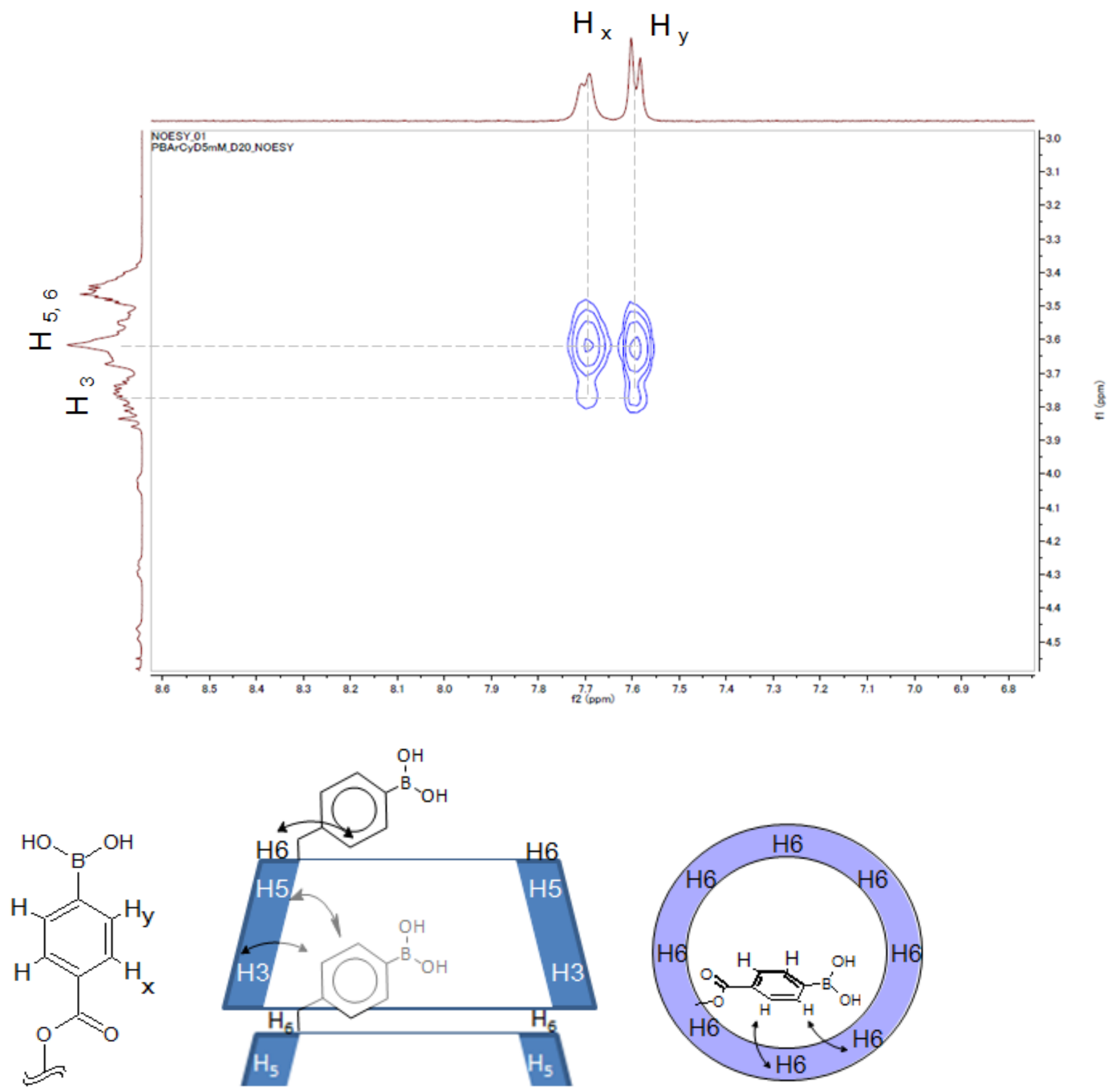

Fig. S10. The ${ }^{1} \mathrm{H}-{ }^{1} \mathrm{H}$ NOESY spectrum of PBA- $\gamma$-CyD $5.0 \mathrm{mM}$ in $\mathrm{D}_{2} \mathrm{O}$ at $25^{\circ} \mathrm{C}$ with mixing time of $200 \mathrm{~ms}$ and its proposed structures. 
(8) PBA- $\gamma-C y D$ in various concentrations

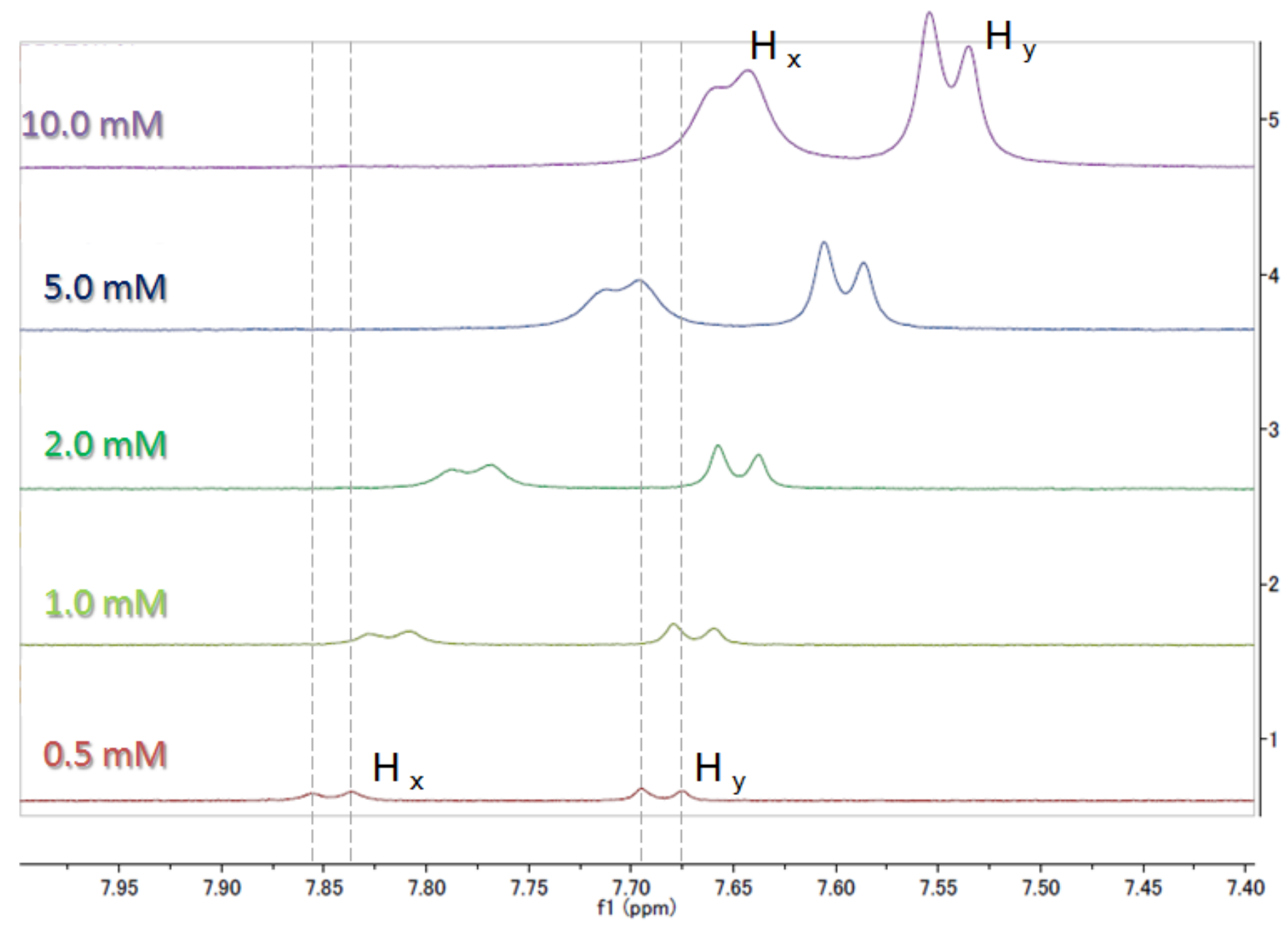

Fig. S11. The ${ }^{1} \mathrm{H}$ NMR spectral changes of PBA moiety depending on the concentration of PBA- $\gamma-\mathrm{CyD}(0.5,1.0$, 2.0, 5.0 and $10.0 \mathrm{mM}$ ) in $\mathrm{D}_{2} \mathrm{O}$ at $25^{\circ} \mathrm{C}$. 
(9) ROESY spectrum of PBA- $\gamma-C y D$ with Naph-PEG

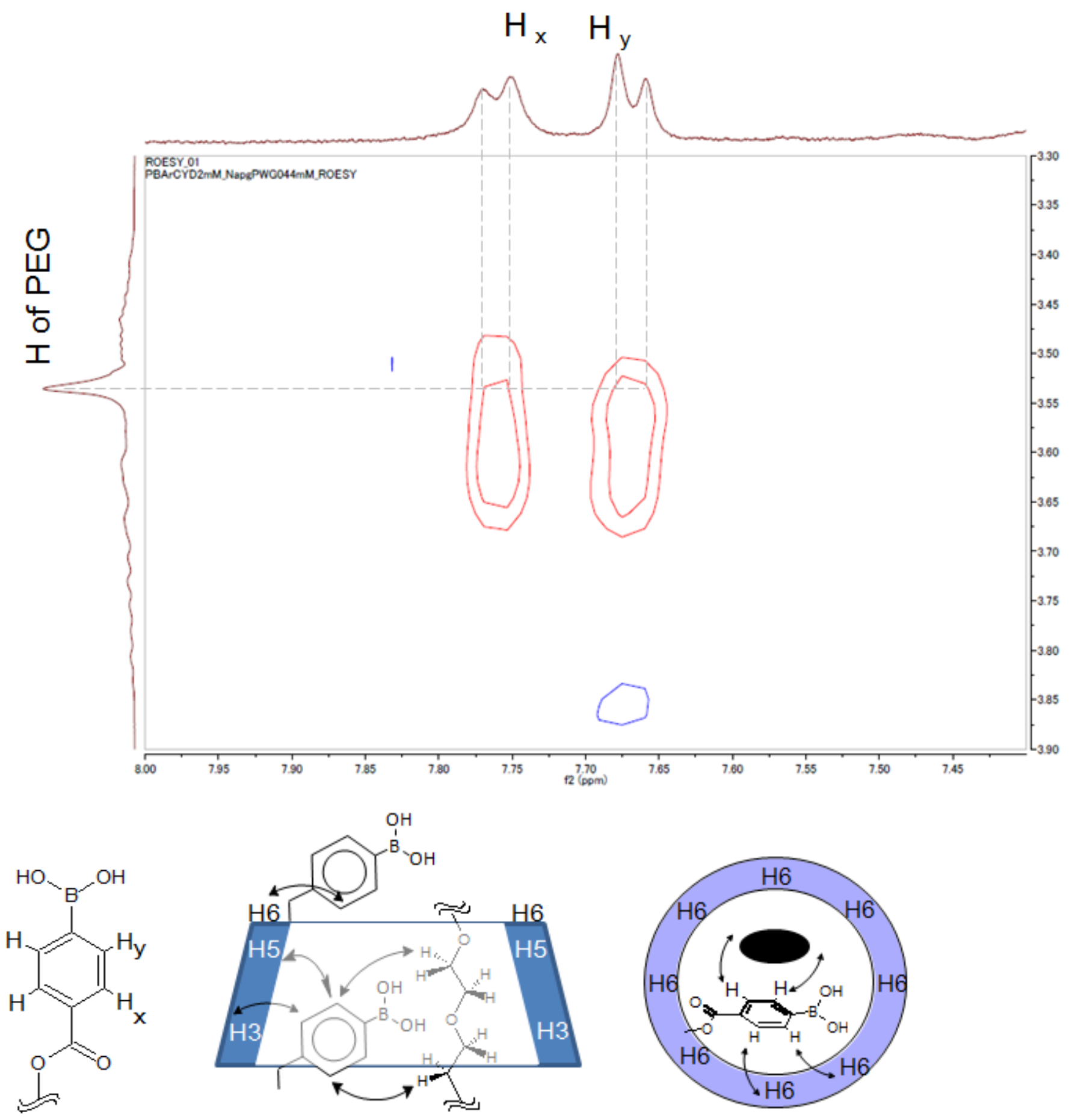

Fig. S12. The ${ }^{1} \mathrm{H}^{1}{ }^{1} \mathrm{H}$ ROESY spectrum of PBA- $\gamma-\mathrm{CyD} 2.0 \mathrm{mM}$ with Naph-PEG $0.4 \mathrm{mM}$ in $\mathrm{D}_{2} \mathrm{O}$ at $25^{\circ} \mathrm{C}$ with mixing time of $200 \mathrm{~ms}$ and its proposed structures. 
2. DSC thermograms

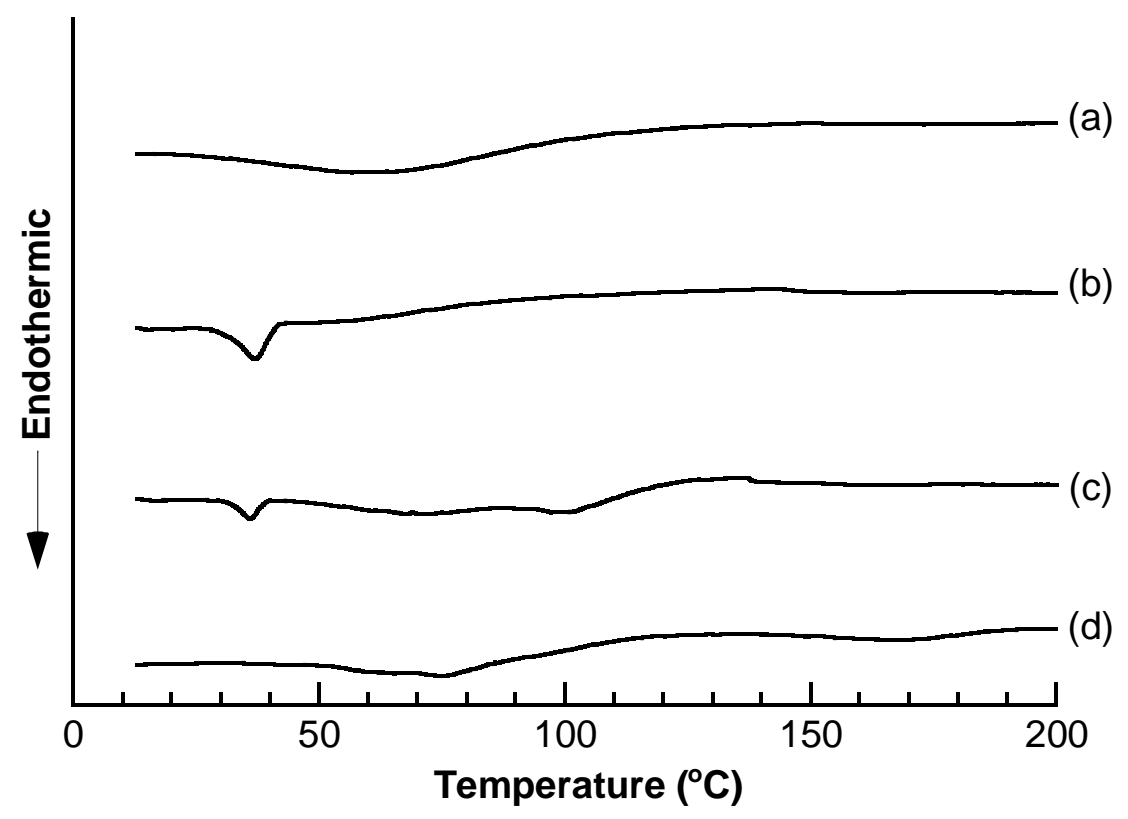

Fig. S13. DSC thermograms: (a) PBA- $\gamma-C y D$, (b) Naph-PEG-Ins, (c) physical mixture of Naph-PEG-Ins and PBA- $\gamma-C y D$, and (d) Naph-PEG-Ins/PBA- $\gamma$-CyD PPRX.

3. XRD patterns of Naph-PEG-Ins/PBA- $\gamma$-CyD PPRX

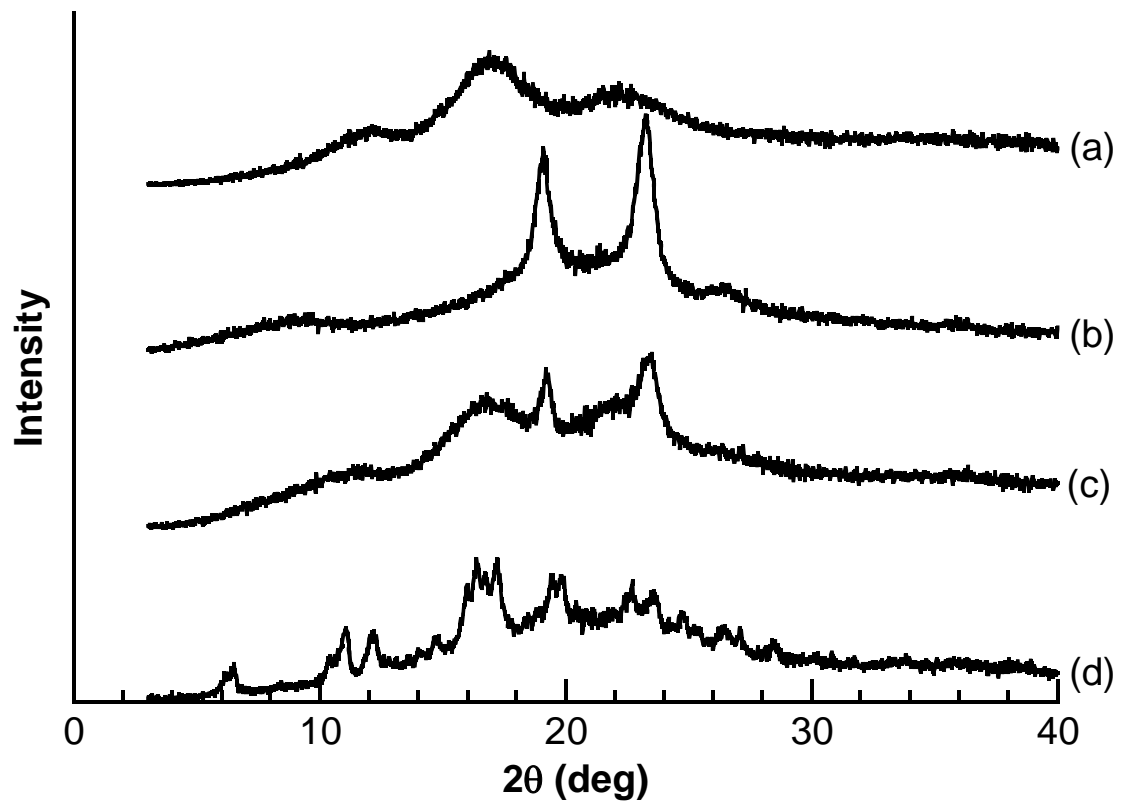

Fig. S14. XRD patterns: (a) PBA- $\gamma$-CyD, (b) Naph-PEG-Ins, (c) physical mixture of Naph-PEG-Ins and PBA- $\gamma$-CyD, and (d) Naph-PEG-Ins/PBA- $\gamma$-CyD PPRX. 